\title{
Dynamics and Control of Worm Epidemic Based on Mobile Networks by SEIQR-Type Model with Saturated Incidence Rate
}

\author{
Rui Hu, ${ }^{1}$ Qingwu Gao ${ }^{D},{ }^{2}$ and Bairong Wang ${ }^{3}$ \\ ${ }^{1}$ Department of Applied Mathematics, Nanjing Audit University, Nanjing, Jiangsu, China \\ ${ }^{2}$ Department of Financial Mathematics, Nanjing Audit University, Nanjing, Jiangsu, China \\ ${ }^{3}$ School of Economics and Management, Shanghai Maritime University, Shanghai, China \\ Correspondence should be addressed to Qingwu Gao; qwgao@aliyun.com
}

Received 12 October 2020; Revised 12 December 2020; Accepted 4 January 2021; Published 18 January 2021

Academic Editor: Maria Alessandra Ragusa

Copyright (c) 2021 Rui Hu et al. This is an open access article distributed under the Creative Commons Attribution License, which permits unrestricted use, distribution, and reproduction in any medium, provided the original work is properly cited.

\begin{abstract}
The mobile networks have increasingly facilitated our daily life but are also breeding grounds for malicious worms, which are considered as the main threat to cyber security. The purpose of this paper is to analyze the dynamics of worm propagation and to control the worm epidemic based on mobile-phone networks. Accordingly, we establish an SEIQR-type model to explore the worm epidemic with saturated incidence rate. This paper shows that if the basic reproduction number is less than 1, the worm-free equilibrium is asymptotically stable, and the epidemic of worm will eventually disappear and remain under control; in addition, if the basic reproduction number is greater than 1, the asymptotical stability of worm-existence equilibrium is derived to imply that the epidemic will remain persistent and uncontrollable. Our results give new insights to mobile network security, namely, that is predicting the worm spreading tendency, identifying the epidemic control strategies, and estimating the worm popularity level. Numerical experiments are conducted to show the rationality of our obtained results and the effectiveness of the control strategies.
\end{abstract}

\section{Introduction}

As everyone knows, the mobile devices, e.g., smartphones, tablets, and laptops, are increasingly pervasive in the world today but have been also both the target and victim of network worm attackers. For example, because of its convenience, the Wi-Fi is currently the most popular way for mobile devices to access to the Internet, which has considerably facilitated our daily life. Unfortunately, it exposes these devices to worm attackers from mobile Internet. A Wi-Fi worm, named Chameleon and appeared in 2014, could spread like an airborne diseases [1]. However, the majority of mobile devices do not have any effective methods to prevent worm attacks. Once a mobile device has been compromised by worms, it can cause great losses to users, including data leakage, system damage, and financial losses [2]. Hence, as considered by network experts, the outbreak of malicious worms is one of the most serious risks to network security, property, and function.
Many researchers have proposed mathematical models to explore the dynamic behaviors of computer worms and biological communities [3-11]. Due to the high similarity between computer worms and biological virus, a series of epidemic models are developed to study the transmission of infectious disease in the host population [12-21]. In the literature mentioned above, we find that the bilinear incidence rate plays an important part in describing the dynamics of virus/worm transmission $[4,22]$. However, in most practical situations, this bilinear rate is impractical so as to considerably restrict the availability of the results obtained. Thus, some nonlinear rates of incidence were introduced by the literature [12, 15, 19-21], among which the saturated rate can tend to its saturation level with the increase of the number of infected or susceptible individuals. Obviously, the saturated rate is more realistic as it can present the inhibition effect of susceptible individuals and the crowding effect of infectious individuals and can also guarantee the boundness of contact rate. In this study, a mathematical model with the saturated rate of incidence is 
taken into consideration to study the worm spreading through mobile networks.

Notice that the above existing models mainly aim at the computer worms spreading through fixed networks, and because of the differences between computers and mobile devices, they could not be applied directly in mobile networks, especially in Wi-Fi ones and 5G ones. Once a mobile device connects to the networks via $\mathrm{Wi}$-Fi, the Wi-Fi base station can control (continue or disrupt) the connection; then, the base station can be adopted to disrupt the connection between the infected devices by worms and other devices. If the connection is cut off, the infected devices are quarantined so that the susceptible devices can be protected from the attack of worms. Hence, in this paper, we consider an SEIQR-type (susceptible-exposed-infectious-quarantined-recovered) model, proposed by Xiao et al. [22], to describe the dynamics and characteristics of the worm propagation based on Wi-Fi networks. Compared with the previous papers, the novel ideas of our paper are that (a) the SEIQR model is along with the saturated rate of incidence; (b) the recovery process from the susceptible group to the recovered group is considered thanks to some sufficient defense mechanisms or antivirus treatments; (c) the dysfunction of devices not only relates to natural death but also depends on worm-related death, because in the real world, the crash of most devices results from the attack of malicious worms; and (d) the worm epidemic intervention strategies are established by reducing its size and speed.

In this paper, we analyze the stability of worm-free and worm-existence equilibria and identify the control strategies of worm epidemic where an optimal strategy is devised to minimize the cost of quarantine intervention and the number of infected devices while maximizing the number of recovered devices. Remark that the worm-free equilibrium stability provides the rationale of worm epidemic intervention strategies; while the worm-existence equilibrium stability can predict the worm spreading tendency for a long period and estimate the worm popularity level by the final scope of infected devices. Furthermore, we carry out a series of numerical experiment in order to demonstrate the rationality of our obtained results and the effectiveness of the control strategies.

The rest of our paper is constructed as follows: in Section 2, we introduce the SEIQR-type model with saturated incidence rate; in Section 3, we analyze the stabilities of worm-free and worm-existence equilibria; in Section 4, we implement the numerical analysis and experiments; in Section 5, we identify the worm epidemic intervention strategies; in Section 6, we conclude and discuss the paper; and in Appendix section, we prove the main results obtained.

\section{SEIQR-Type Worm Propagation Model}

In the paper, we consider the general SEIQR-type worm propagation model, in which the mobile devices are divided into five groups: susceptible group $(S)$ includes the devices uninfected by malicious worms but vulnerable to worm attacks; exposed group $(E)$ includes the devices exposed to and infected by the worms but not infectious; infectious group (I) includes the exposed and infectious devices; quarantine group $(Q)$ includes the infectious and quarantined devices; recovered group $(R)$ includes the recovered devices with immunity against the worms. Figure 1 shows the state transition diagram with the involved modeling parameters, Table 1 lists the baseline parameters and their initial values, and Table 2 lists the state transitions and their rates.

As shown in Figure 1 and Tables 1 and 2, we present the state transitions of the SEIQR-type model in detail as follows:

(1) Since the susceptible devices are of no immunity against malicious worms, they can transit to the exposed group at the saturated incidence rate $\beta S I /(1+\alpha I)$. Note that $\beta I /(1+\alpha I)$ goes to its saturation level as $I$ increases, $\beta I$ denotes the force of worm infection, and $1 /(1+\alpha I)$ denotes the crowding effect of infectious ones and guarantees the boundness of contact rate by an appropriate value for $\alpha$.

(2) The exposed devices become infectious at rate $\eta$, where $1 / \eta$ denotes the mean value of latent period. If .., the latent period tends to 0 , which follows that the SEIQR model can reduce to a SIQR one.

(3) Due to the quarantine intervention implemented on the infectious group, the infectious ones can transit to the quarantined group at rate $\xi$. As $\xi=0$, there is no quarantine intervention, and then the SEIQR model reduces to a SEIR one [13, 19].

(4) Thanks to some sufficient defense mechanisms or antivirus treatments, the susceptible, exposed, infectious, and quarantined devices transit to the recovered group at rates $\psi, \varepsilon, \gamma$, and $\varphi$, respectively. Note that $1 / \varphi$ denotes the mean value of quarantine period. If $\varphi \longrightarrow \infty$, then the quarantine period tends to 0 , which follows that the SEIQR model reduces to an SEIR one $[13,19]$.

(5) The dysfunction of mobile device can take place in all groups due to not only the natural death but also the worm-related death. The dysfunction replacement is taken to the susceptible group at rate $\Lambda$.

Therefore, the general SEIQR-type model considered in the paper is formulated by the following system of nonlinear ordinary differential equations:

$$
\begin{aligned}
S^{\prime}(t) & =\Lambda-\frac{\beta S(t) I(t)}{1+\alpha I(t)}-(\psi+\mu) S(t), \\
E^{\prime}(t) & =\frac{\beta S(t) I(t)}{1+\alpha I(t)}-(\eta+\varepsilon+\mu) E(t), \\
I^{\prime}(t) & =\eta E(t)-(\xi+\gamma+\mu+\theta) I(t), \\
Q^{\prime}(t) & =\xi I(t)-(\varphi+\mu+\theta) Q(t), \\
R^{\prime}(t) & =\psi S(t)+\varepsilon E(t)+\gamma I(t)+\varphi Q(t)-\mu R(t) .
\end{aligned}
$$




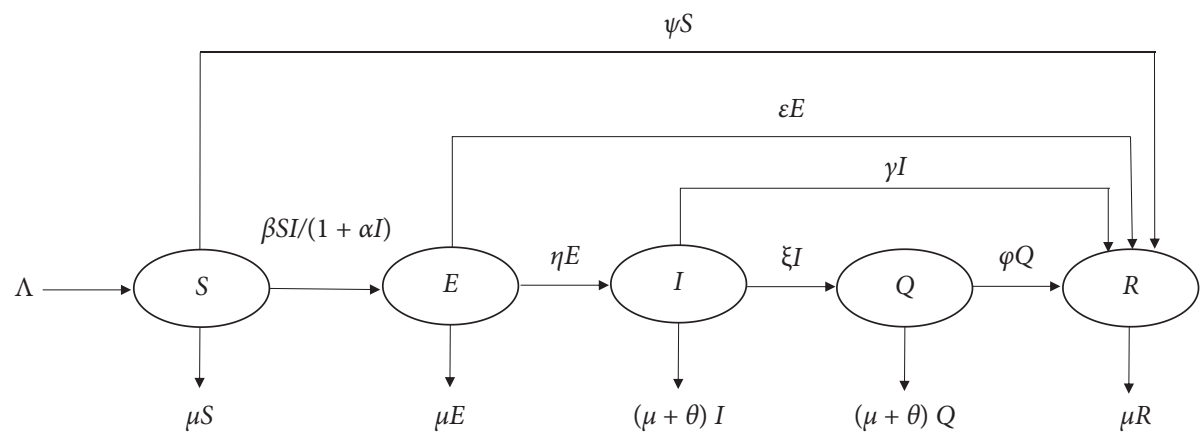

FIGURE 1: State transition diagram of the SEIQR-type model.

The total number of mobile devices at time $t, N(t)$, satisfies that

$$
N(t)=S(t)+E(t)+I(t)+Q(t)+R(t) .
$$

By a summation of all the equations of system (1), we have

$$
N^{\prime}(t) \leq \Lambda-\mu N(t)
$$

which implies that

$$
\begin{aligned}
N(t) & \leq \frac{\Lambda}{\mu}+\left[N(0)-\frac{\Lambda}{\mu}\right] e^{-\mu t}, \\
\lim _{t \rightarrow \infty} N(t) & =\frac{\Lambda}{\mu} .
\end{aligned}
$$

Hence, we define system (1)'s feasible region as

$$
\Omega=\left\{(S, E, I, Q, R) \in \mathbb{R}^{5} \mid 0 \leq S+E+I+Q+R \leq \frac{\Lambda}{\mu}\right\} .
$$

Now, we make a nonnegativity analysis to ensure the plausibility of SEIQR-type model (1). By the first equation in (1), we have

$$
S^{\prime}(t) \leq \Lambda-(\psi+\mu) S(t)
$$

which implies that

$$
S(t) \leq \frac{\Lambda}{\psi+\mu}+\left[S(0)-\frac{\Lambda}{\psi+\mu}\right] e^{-(\psi+\mu) t},
$$

and then in a long run,

$$
S(t) \leq \frac{\Lambda}{\psi+\mu}=: \Delta_{1} .
$$

Further by the second equation in (1), we have

$$
E^{\prime}(t) \leq \frac{\beta \Delta_{1}}{\alpha}-(\eta+\varepsilon+\mu) E(t)
$$

which implies that

$$
E(t) \leq \frac{\beta \Delta_{1}}{\alpha(\eta+\varepsilon+\mu)}+\left[E(0)-\frac{\beta \Delta_{1}}{\alpha(\eta+\varepsilon+\mu)}\right] e^{-(\eta+\varepsilon+\mu) t},
$$

TABLE 1: Explanations and initial values of modeling parameter.

\begin{tabular}{lcc}
\hline Parameters & Explanations & Initial values \\
\hline$N(t)$ & Device number at time $t$ & $N(0)=75,000$ \\
$S(t)$ & Number of susceptible devices at time $t$ & Not fixed \\
$E(t)$ & Number of exposed devices at time $t$ & Not fixed \\
$I(t)$ & Number of infectious devices at time $t$ & Not fixed \\
$Q(t)$ & Number of quarantined devices at time $t$ & $Q(0)=0$ \\
$R(t)$ & Number of recovered devices at time $t$ & $S(0)=0$ \\
$\Lambda$ & Rate of recruitment & 0.75 \\
$\mu$ & Rate of natural death & 0.00001 \\
$\theta$ & Rate of worm-related death & 0.001 \\
$\alpha$ & Parameter of saturated incidence rate & 0.8 \\
$\beta$ & Infection rate in group $S$ & 0.053 \\
$\psi$ & Rate of transition from $S$ to $R$ & Not fixed \\
$\eta$ & Rate of transition from $E$ to $I$ & 0.008 \\
$\varepsilon$ & Rate of transition from $E$ to $R$ & 0.0008 \\
$\gamma$ & Rate of transition from $I$ to $R$ & 0.05 \\
$\xi$ & Rate of transition from $I$ to $Q$ & 0.05 \\
$\varphi$ & Rate of transition from $Q$ to $R$ & 0.005 \\
\hline
\end{tabular}

TABLE 2: State transition events and transition rates.

\begin{tabular}{lc}
\hline Transition events & Rate of transition \\
\hline$S \longrightarrow E$ & $\beta S(t) I(t) /(1+\alpha I(t))$ \\
$S \longrightarrow R$ & $\psi S(t)$ \\
$E \longrightarrow I$ & $\eta E(t)$ \\
$E \longrightarrow R$ & $\varepsilon E(t)$ \\
$I \longrightarrow Q$ & $\xi I(t)$ \\
$Q \longrightarrow R$ & $\varphi Q(t)$ \\
$S \longrightarrow$ Dysfunction & $\mu S(t)$ \\
$E \longrightarrow$ Dysfunction & $\mu E(t)$ \\
$I \longrightarrow$ Dysfunction & $(\theta+\mu) I(t)$ \\
$Q \longrightarrow$ Dysfunction & $(\theta+\mu) Q(t)$ \\
$R \longrightarrow$ Dysfunction & $\mu R(t)$ \\
Recruitment $\longrightarrow S$ & $\Lambda$ \\
\hline
\end{tabular}

and then in a long run,

$$
E(t) \leq \frac{\beta \Delta_{1}}{\alpha(\eta+\varepsilon+\mu)}=: \Delta_{2}
$$

Arguing as above, it follows that 


$$
\begin{aligned}
& I(t) \leq \frac{\eta \Delta_{2}}{\xi+\gamma+\mu+\theta}=\Delta_{3}, \\
& Q(t) \leq \frac{\xi \Delta_{3}}{\varphi+\mu+\theta}=\Delta_{4}, \\
& R(t) \leq \frac{\psi \Delta_{1}+\varepsilon \Delta_{2}+\gamma \Delta_{3}+\varphi \Delta_{4}}{\mu} .
\end{aligned}
$$

Thus, by the above results, we get a proposition below.

Proposition 1. System (1) has solutions satisfying $S(t)>0$, $E(t)>0, I(t)>0, Q(t)>0$, and $R(t)>0$ for all $t>0$, and its feasible region $\Omega$ is an attracting and positively invariant set.

\section{Dynamics Analysis}

In this section, we analyze the stabilities of worm-free and worm-existence equilibria. Firstly, we calculate the equilibrium points and the basic reproduction number. Secondly, we study the stability of worm-free equilibrium which can establish worm epidemic intervention strategies. Thirdly, we present the system uniform persistence. Finally, we study the stability of worm-existence equilibrium which can predict the worm spreading tendency and estimate the epidemic popularity level.

3.1. Basic Reproduction Number and Equilibrium Points. To analyze the dynamics of the SEIQR-type model described by (1), we determine the equilibria satisfying

$$
\left\{\begin{array}{l}
S^{\prime}(t)=0 \\
E^{\prime}(t)=0 \\
I^{\prime}(t)=0 \\
Q^{\prime}(t)=0 \\
R^{\prime}(t)=0
\end{array}\right.
$$

By $I^{\prime}(t)=0$, it follows that

$$
E=\frac{(\xi+\gamma+\mu+\theta) I}{\eta},
$$

which is substituted into $E^{\prime}(t)=0$ to get that

$$
\left[\frac{\beta S}{1+\alpha I}-\frac{(\eta+\varepsilon+\mu)(\xi+\gamma+\mu+\theta)}{\eta}\right] I=0 .
$$

Hence, we have

$$
I=0 \text {, }
$$

$$
\text { or } I>0 \text {, }
$$

$$
S=\frac{(\eta+\varepsilon+\mu)(\xi+\gamma+\mu+\theta)(1+\alpha I)}{\beta \eta} .
$$

If $I=0$, we obtain the unique worm-free equilibrium point of system (1) as

$$
P^{0}=\left(S^{0}, E^{0}, I^{0}, Q^{0}, R^{0}\right)=\left(\frac{\Lambda}{\psi+\mu}, 0,0,0, \frac{\psi}{\mu} S^{0}\right) .
$$

If $I>0$, we derive by substituting $S$ in (16) into $S^{\prime}(t)=0$ that

$$
\begin{aligned}
& (\eta+\varepsilon+\mu)(\xi+\gamma+\mu+\theta)[\beta+\alpha(\psi+\mu)] \\
& I-[\beta \eta \Lambda-(\psi+\mu)(\eta+\varepsilon+\mu)(\xi+\gamma+\mu+\theta)]=0 .
\end{aligned}
$$

Define the basic reproduction number $R_{0}$ as

$$
R_{0}=\frac{\beta \eta \Lambda}{(\psi+\mu)(\eta+\varepsilon+\mu)(\xi+\gamma+\mu+\theta)} .
$$

Clearly, if $R_{0} \leq 1$, the worm-free equilibrium is the unique equilibrium; if $R_{0}>1$, the worm-existence equilibrium is the unique positive equilibrium $P^{*}=\left(S^{*}, E^{*}, I^{*}\right.$, $\left.Q^{*}, R^{*}\right)$, where

$$
I^{*}=\frac{\beta \eta \Lambda-(\psi+\mu)(\eta+\varepsilon+\mu)(\xi+\gamma+\mu+\theta)}{(\eta+\varepsilon+\mu)(\xi+\gamma+\mu+\theta)[\beta+\alpha(\psi+\mu)]},
$$

and then $S^{*}, E^{*}, Q^{*}$, and $R^{*}$ satisfy

$$
\left\{\begin{array}{l}
S^{*}=\frac{(\eta+\varepsilon+\mu)(\xi+\gamma+\mu+\theta)\left(1+\alpha I^{*}\right)}{\beta \eta}, \\
E^{*}=\frac{(\xi+\gamma+\mu+\theta) I^{*}}{\eta}, \\
Q^{*}=\frac{\xi I^{*}}{\varphi+\mu+\theta}, \\
R^{*}=\frac{\psi S^{*}+\varepsilon E^{*}+\gamma I^{*}+\varphi Q^{*}}{\mu} .
\end{array}\right.
$$

Hence, we give the following result regarding the equilibrium points.

Theorem 1. If $R_{0} \leq 1$, the worm-free equilibrium $P^{0}$ is the unique equilibrium point satisfying (17); if $R_{0}>1$, the wormexistence equilibrium $P^{*}$ is the unique positive equilibrium point satisfying (20) and (21).

Remark 1. The worm-existence equilibrium point $\left(S^{*}, E^{*}, I^{*}, Q^{*}\right.$, and $\left.R^{*}\right)$ can access the final scales of mobile devices in all the groups for a long period. Especially, the final scale of infected devices, namely, $E^{*}+I^{*}+Q^{*}$, can estimate the popularity level of worm epidemic.

In fact, the basic reproduction number $R_{0}$ is an important concept in virus/worm epidemic dynamics, which is "the average number of secondary cases generated by one primary case at the start of the epidemic in a completely susceptible population" $[23,24]$ and can also be obtained by the next generation matrix method [23] or the next generation operator theory [24]. From Theorem 1, the sharp threshold value, $R_{0}$, can completely determine the existence of two equilibrium points. 
3.2. Stability of Worm-Free Equilibrium. In this section, we study the local and global stabilities for worm-free equilibrium $P^{0}$, and their proofs can be found in Appendixes A.1 and A.2.

Theorem 2. If $R_{0} \leq 1$, the worm-free equilibrium $P^{0}$ is locally asymptotically stable. Otherwise, $P^{0}$ is an unstable point.

Theorem 3. If $R_{0} \leq 1$, the worm-free equilibrium $P^{0}$ is globally asymptotically stable. Otherwise, $P^{0}$ is an unstable point.

Combining Theorems 2 and 3, we know that the local and global stabilities of worm-free equilibrium are equivalent.

Remark 2. From Theorem 2 and 3, we conclude that if $R_{0} \leq 1$, the exposed, infectious, and quarantined devices all tend to 0 , namely, that the infected devices tend to 0 . Accordingly, $R_{0} \leq 1$ indicates that the epidemic of worm will eventually disappear and remain under control, and then to prevent and control the epidemic, some related intervention strategies should be adopted to make $R_{0}$ smaller than 1 , and see those in Section 5. Clearly, Theorems 2 and 3 provide the theoretical basis of those worm epidemic intervention strategies.

We find out from Theorems 2 and 3 and Remark 2 that the basic reproduction number $R_{0}$, as a sharp threshold value, will play a critical role to control the worm epidemic based on mobile networks, because the threshold value $R_{0}$ can completely determine the local and global dynamics for worm-free equilibrium point $P^{0}$ and also can govern if the mobile devices infected by worms become extinct for time locally and globally.

3.3. Uniform Worm Persistence. With the use of the acyclicity theorem [25], we present the uniform persistence of system (1) if $R_{0}>1$, whose proof is given in Appendix A.3.

Definition 1 (see [26]). System (1) is uniformly persistent in $\Omega$, if there is $c \in(0,1)$ such that any solution $(S(t), E(t), I(t), Q(t)$, and $R(t))$ of (1) with initial value satisfies

$$
\min \left\{\liminf _{t \longrightarrow \infty} S(t), \liminf _{t \longrightarrow \infty} E(t), \liminf _{t \longrightarrow \infty} I(t), \liminf _{t \longrightarrow \infty} Q(t), \liminf _{t \longrightarrow \infty} R(t)\right\} \geq c
$$

Theorem 4. If $R_{0}>1$, the system (1) is uniform persistent in the bounded set $\Omega$.

Remark 3. The uniform persistence of (1) in $\Omega$ means that there exists a compact set $K \subset \Omega$ which is absorbent for system (1) (see [27]).

3.4. Stability of Worm-Existence Equilibrium. We now perform the stability analysis for the worm-existence equilibrium $P^{*}$ and prove its related main results in Appendixes A.4 and A.5. From an epidemiological point of view; $P^{*}$ is more necessary to study than its counterpart, i.e., the worm-free equilibrium $P^{0}$.

Theorem 5. If $R_{0}>1$, the worm-existence equilibrium $P^{*}$ is locally asymptotically stable.

It is well-known that the local stability for $P^{*}$ may be insignificant in practice, because it only assures the stability of microvariations relative to its initial state of $P^{*}$. Hence, we turn to the global stability for $P^{*}$ by the Li-Muldowney geometric approach [28].

Theorem 6. If $R_{0}>1$, the worm-existence equilibrium $P^{*}$ is globally asymptotically stable.
Remark 4. The global stability for $P^{*} \in \operatorname{int}(\Omega)$ is equivalent to that the set $\Omega-\{(S, E, I, Q, R) \mid I t=n 0\}$ is a globally stable set for $P^{*}$.

Remark 5. From Theorem 5 and 6 , the stability for $P^{*}$ shows that if $R_{0}>1$, all the groups of mobile devices will tend to $S^{*}$, $E^{*}, I^{*}, Q^{*}$, and $R^{*}$, respectively, which are those in $(20)$ and (21). Accordingly, we conclude that $R_{0}>1$ assures that the epidemic of mobile network-based worm remains persistent and uncontrollable, where we can predict the worm spreading tendency in a long run and access the epidemic popularity level by the final scope of infected devices (i.e., $\left.E^{*}+I^{*}+Q^{*}\right)$.

From the arguments of Theorem 5 and 6 and Remark 5, we also see that the basic reproduction number $R_{0}$ is an exact threshold value which guarantees the local and global stabilities for $P^{*}$ and governs whether the network-based worm keeps always transmitting locally and globally.

\section{Performance Evaluation}

In this section, we carry out numerical simulations to verify the feasibility of the dynamics of worm propagation via the SEIQR-type model.

4.1. Simulation Setting. In the realistic scenario, it is quite hard for researchers to get the corresponding data of worm traffic traces. Even for some available data from MIT or CAIDA (http://www.caida.org), the legitimate flow of traffic 
traces is not contained since these traces have been intentionally filtered out before they are available. Hence, we could not easily determine the necessary and appropriate parameters of the constructed SEIQR-type model. However, in the paper, we plan to choose the same parameter values as those in [29] to fit a real worm epidemic based on mobile networks.

As a typical example, we take Slammer worm in this experiment. As one of the fastest spreading worms, Slammer had attacked 75,000 devices at the early stage after its outbreak in January 2003 [30]. Thus, the total number of devices is set to be 75,000 . The infection rate $\beta$ is a key parameter that determines the spreading speed of worms among mobile devices. Consider that Slammer is a bandwidth-limited worm with an average scan rate $s=4,000$ scans/second [31]; then, by the same method in [32], we get the worm-infection rate as

$$
\beta=\frac{s}{N} \approx 0.053 \text {. }
$$

The rate of natural death is $\mu=0.00001$, and the rate of worm-related death is $\theta=0.001$. The rates of state transitions from $E$ to $I$, from $I$ to $R$, and from $Q$ to $R$ are $\eta=0.008$, $\gamma=0.05$, and $\varphi=0.005$, respectively. These parameters come from the average value of more than 10 simulation operations [31].

The rest parameters involved of the SEIQR-type model are determined as follows. According to the definition of region $\Omega$, the recruitment rate is $\Lambda=0.75$. Given the quarantine intervention on the group of infectious devices, the quarantine rate is $\xi=0.05$ [22]. Based on the fact that the average value of recovery period from the exposed group is approximately 1, 200 seconds [7], we get the rate of recovery from group $E$ to group $R$ as

$$
\varepsilon=\frac{1}{1200} \approx 0.0008 \text {. }
$$

Due to one or more security countermeasures which have been taken initially for the susceptible devices to prevent the worm attack, the recovery rate from states $\mathrm{S}$ to $R$ is set to $\psi=0.5$. Finally, we set the parameter $\alpha$ of the saturated incidence rate as 0.8 [29].

\subsection{Sensitivity Analysis for Parameter $\alpha$ of Saturated Incidence} Rate. In this section, we make a sensitivity analysis for the constant parameter $\alpha$ of the saturated incidence rate by a numerical experiment, where we choose $\alpha=0,0.8$, and 2 , and the rest parameters involved as the same as the ones in Section 4.1. Please see Figure 2, which gives the impact of $\alpha$ on the numbers of infectious and recovered devices. More specifically, we observe from Figure 2(a) that as the parameter $\alpha$ increases, the number of infectious devices decreases and the duration of worm epidemic becomes shorter (see Figure 2(a)), and the number of recovered devices increases at a higher speed (see Figure 2(b)), which shows that increasing the parameter $\alpha$ can be taken as an effectively approach to control the worm epidemic based on mobile networks.
Especially, if the parameter $\alpha=0$, in other words, the SEIQR-type model considered is accompanied by a bilinear incidence rate rather than a saturated incidence rate, we know that by Figure 2 that the mobile network-based worm can infect much more devices and its epidemic keeps ongoing for a longer period, and the devices becoming recovered from all groups are less and reach their maximum size in an even longer period. Obviously, the saturated rate is more effective and reasonable than the bilinear rate to control the worm epidemic based on mobile networks.

\subsection{Dynamics of Worm-Free and Worm-Existence Equilibria.} In this section, we present simulation results to describe the tendency of worm propagation and then to validate the plausibility of the obtained results.

Firstly, we set the initial numbers in all device groups as $S(0)=45000, E(0)=20000, I(0)=10000, Q(0)=0$, and $R(0)=0$, while the modeling parameters as the same as those in Section 4.1. Then, using equation (17), $P^{0}=(1.5,0,0,0,74998.5)$, and using equation (19), $R_{0}=0.715<1$. Based on Theorems 2 and $3, P^{0}$ is locally and globally asymptotically stable, respectively, implying that as $t \longrightarrow \infty, S(t)$, and $R(t)$ will tend to steady states 1.5 and 74998.5, while $E(t), I(t)$, and $E(t)$ will all tend to 0 . Hence, the final size of infected devices, $E^{0}+I^{0}+Q^{0}$, is 0 , which tell us that the worm will gradually become extinct and its epidemic remain under control in the end. As seen in Figure 3, the worm propagation tendency is fairly depressive for a long period, and the final scope of infected devices will vanish eventually, which is in accordance with the results of Theorems 2 and 3.

Secondly, all the parameters and initial numbers are set as those in the first part of this section except for $\psi=0.05$. By equations (20) and (21), $P^{*}=(7.64,41.67,3.3,27.45$, 71758.6 ), and by equation (19), $R_{0}=7.146>1$. Accordingly, we conclude from Theorems 5 and 6 that $P^{*}$ is locally and globally stable, namely, that as $t \longrightarrow \infty, S(t), E(t), I(t)$, $Q(t)$, and $R(t)$ will tend to $7.64,41.67,3.3,27.45$, and 71758.6, respectively. Please see Figure 4, which gives the worm propagation trend for a long period. Particularly, we can get the final size of the infected devices, i.e., $E^{*}+I^{*}+Q^{*}=72.42$, to evaluate the worm epidemic popularity level. In other words, the worm epidemic remains always propagating within a population of about 72.42 . From Figure 4(a), we get the rough curves of $S(t), E(t), I(t), Q(t)$, and $R(t)$, and further from Figure 4(b), we get the tendency of $S(t), E(t), I(t)$, and $Q(t)$ in more detail. The numerical results of Figure 4 are in accordance with Theorems 5 and 6 .

Thirdly, as shown in Figures 3 and 4, the infected devices will eventually vanish or approach to a negligible size, and nearly all groups of devices will get recovered in the end, which suggests that the worm epidemic does exist in mobile networks but be of negligible scale owing to the quarantine strategy and other measures taken to the susceptible and infected devices. Consequently, these simulations validate the proposed SEIQR-type model in defending against malicious worms. 


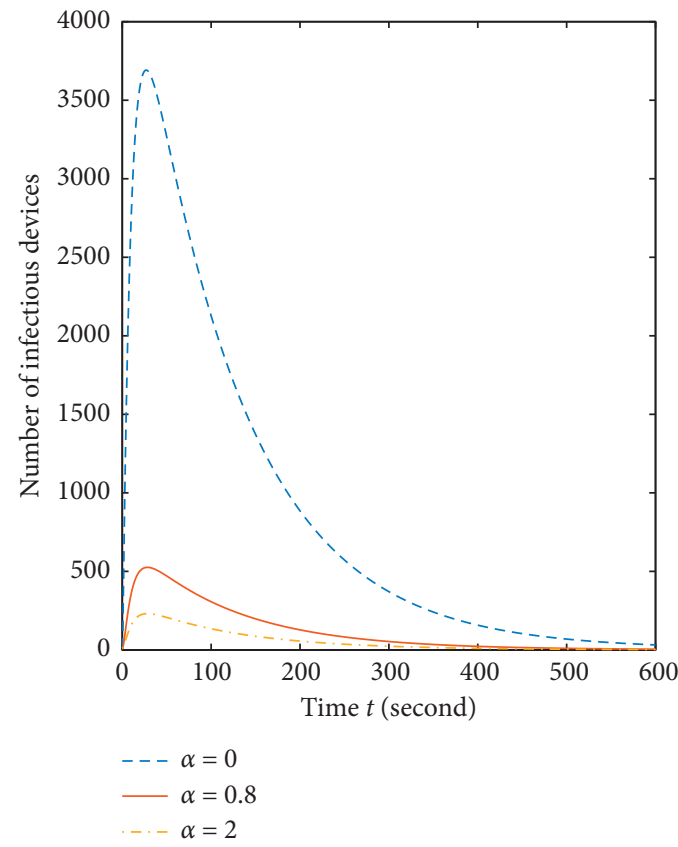

(a)

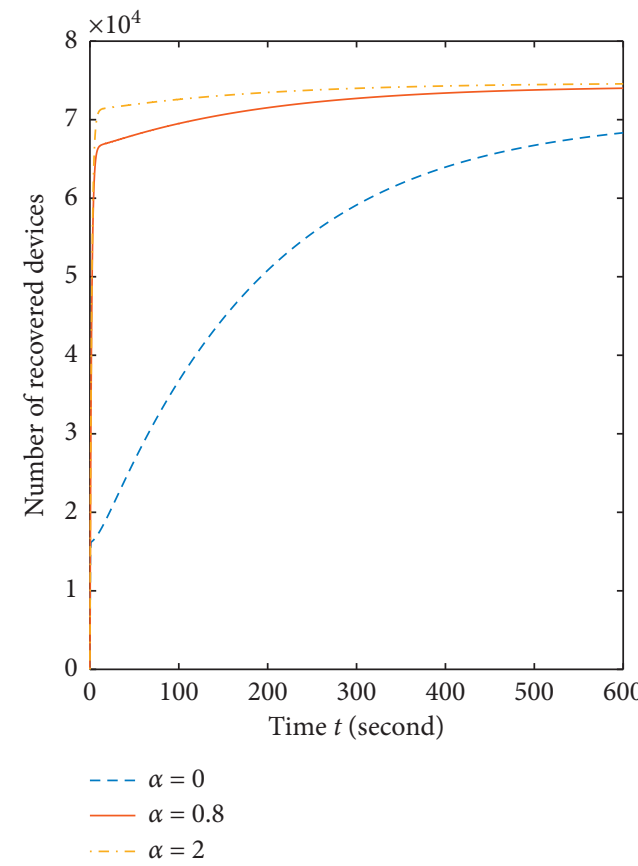

(b)

FIGURE 2: Sensitivity analysis of parameter in saturated incidence rate.

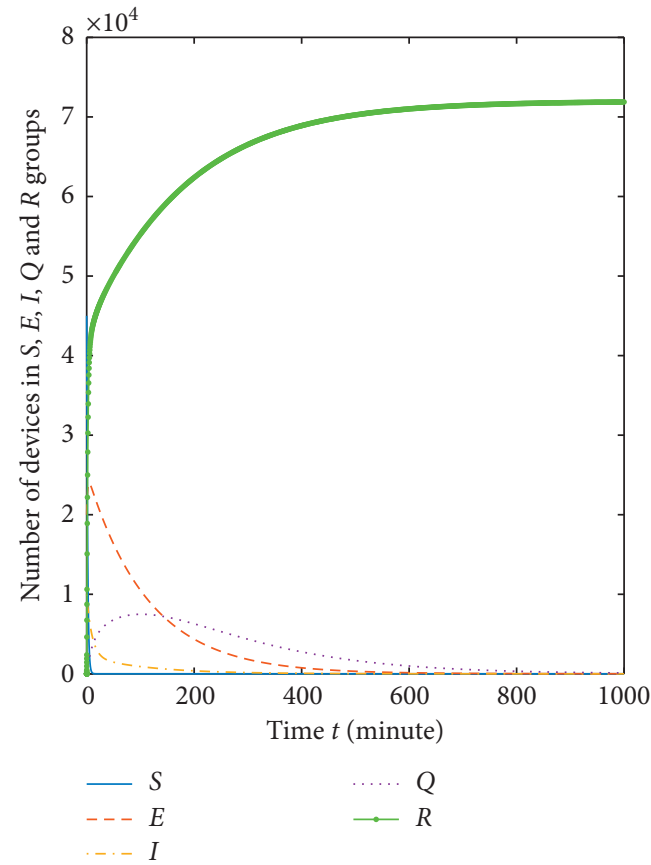

(a)

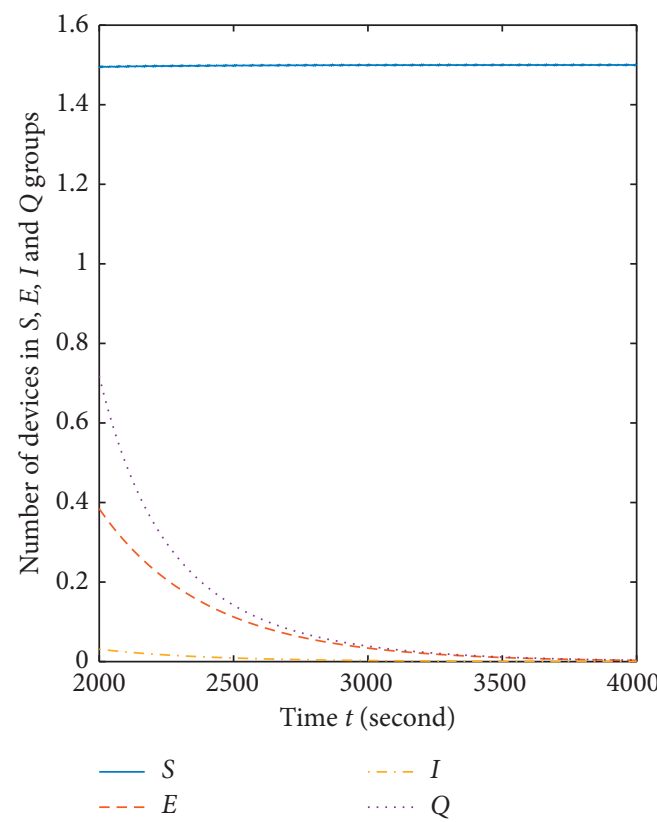

(b)

Figure 3: Dynamics of the worm-free equilibrium.

\section{Control Strategies on Worm Epidemic}

To control biological viruses and network worms, the optimal control strategy is a frequently-used mathematical approach [33], and its related numerical simulations and region plots have also been considered in tails [33, 34]. So in what follows, we firstly construct the optimal control strategy based on Pontryagin's Minimum Principle [35] to minimize the cost of quarantine intervention and the 


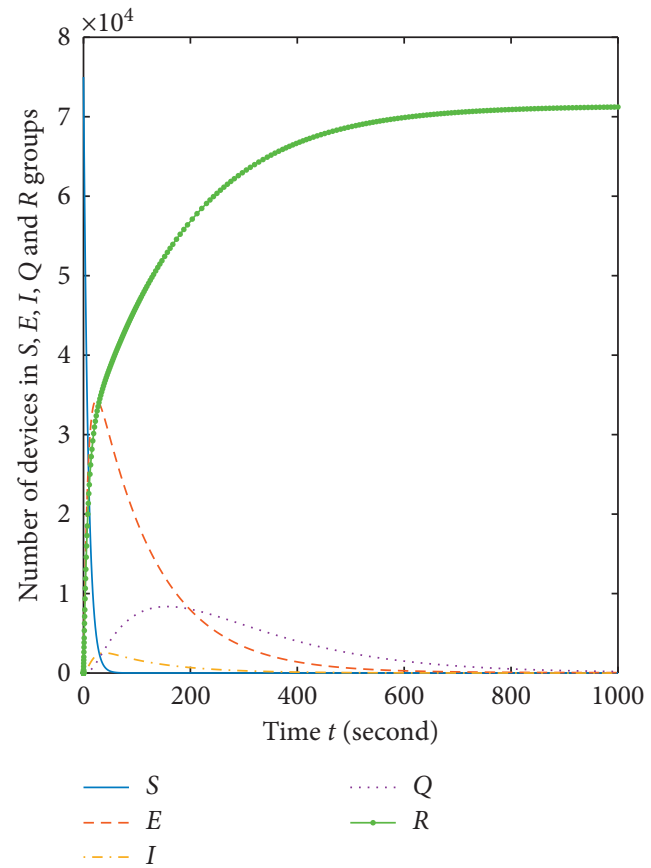

(a)

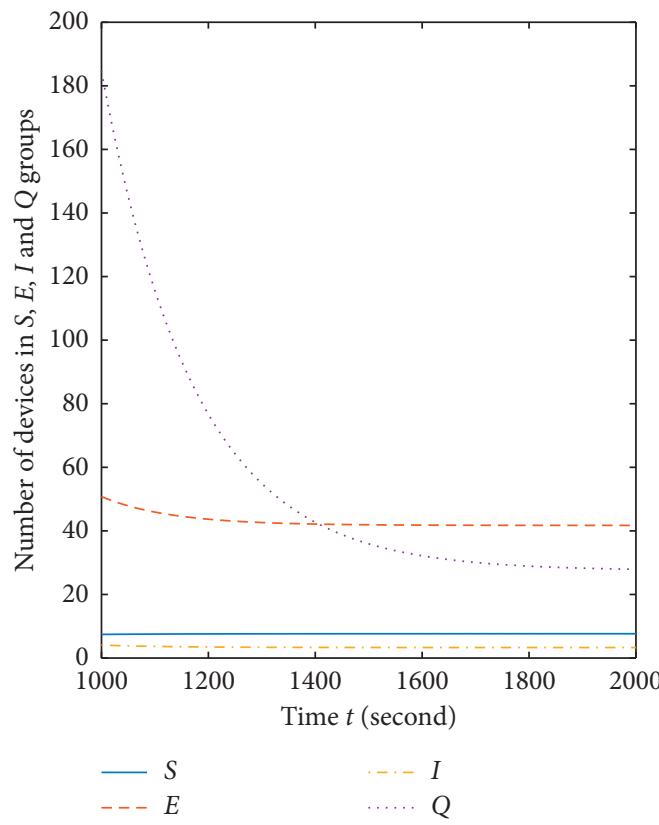

(b)

FIgURe 4: Dynamics of worm-existence equilibrium.

number of infected devices as well as maximize the number of recovered devices, and secondly we establish other control strategies with the use of Theorems 2 and 3 to control the worm epidemic based on mobile networks. Besides, a series of corresponding numerical simulations are implemented to validate the effectiveness of control strategies mentioned above.

5.1. The Optimal Control Strategy. In this section, the optimal control strategy is constructed for the SEIQR-type model described by system (1), where a control variable $u(t)$, $0 \leq u(t) \leq 1$, is chosen to denote the inhibiting effect of quarantine strategy on the worm epidemic. Especially, when $u(t)=1$, the quarantine strategy is perfectly effective; when $u(t)=0$, the quarantine strategy has no effect at all. Hence, the optimal control problem is formulated as

$$
J(u)=\int_{t_{0}}^{t_{f}}\left[K u^{2}+E(t)+I(t)-R(t)\right] \mathrm{d} t,
$$

where the parameter $K \geq 0$ denotes the weight of the benefit of quarantine cost, and this problem is subject to the following state system:

$$
\left\{\begin{array}{l}
S^{\prime}(t)=\Lambda-\frac{\beta S(t) I(t)}{1+\alpha I(t)}-(\psi+\mu) S(t), \\
E^{\prime}(t)=\frac{\beta S(t) I(t)}{1+\alpha I(t)}-(\eta+\varepsilon+\mu) E(t), \\
I^{\prime}(t)=\eta E(t)-(u(t)+\gamma+\mu+\theta) I(t), \\
Q^{\prime}(t)=u(t) I(t)-(\varphi+\mu+\theta) Q(t), \\
R^{\prime}(t)=\psi S(t)+\varepsilon E(t)+\gamma I(t)+\varphi Q(t)-\mu R(t) .
\end{array}\right.
$$

The main goal of optimal control is to determine the control variable $u^{*}$ satisfying 


$$
J\left(u^{*}\right)=\min \{J(u): u \in U\}
$$

where $U$ is the control set defined by $U=\left\{u(t): u(t)\right.$ is measurable, $\left.0 \leq u(t) \leq 1, t \in\left[t_{0}, t_{f}\right]\right\}$.

According to Fleming and Rishel (1975, [36]), we now consider the existence of the optimal control variable $u^{*}$.
Theorem 7. The optimal control variable $u^{*} \in U$ does exist such that (27) holds under its state system (26) with initial conditions at $t_{0}$.

The Hamiltonian for state system (26) is characterized as

$$
\begin{aligned}
H= & K u^{2}+E+I-R+w_{1}\left[\Lambda-\frac{\beta S I}{1+\alpha I}-(\psi+\mu) S\right]+w_{2}\left[\frac{\beta S I}{1+\alpha I}-(\eta+\varepsilon+\mu) E\right] \\
& +w_{3}[\eta E-(u+\gamma+\mu+\theta) I]+w_{4}[u I-(\varphi+\mu+\theta) Q]+w_{5}[\psi S+\varepsilon E+\gamma I+\varphi Q-\mu R],
\end{aligned}
$$

which, along with the Pontryagin's Minimum Principle [35], leads to a theorem below.
Theorem 8. Given an optimal control variable $u$ and corresponding solution of state system (26), there are adjoint variables $w_{i}, i=1, \ldots, 5$, such that the following adjoint system of equations holds:

$$
\left\{\begin{array}{l}
w_{1}^{\prime}(t)=-\frac{\partial H}{\partial S}=\frac{\left(w_{1}-w_{2}\right) \beta I}{1+\alpha I}+w_{1} \mu+\left(w_{1}-w_{5}\right) \psi \\
w_{2}^{\prime}(t)=-\frac{\partial H}{\partial E}=-1+\left(w_{2}-w_{3}\right) \eta+\left(w_{2}-w_{5}\right) \varepsilon+w_{2} \mu \\
w_{3}^{\prime}(t)=-\frac{\partial H}{\partial I}=-1+\frac{\left(w_{1}-w_{2}\right) \beta S}{(1+\alpha I)^{2}}+\left(w_{3}-w_{4}\right) u+\left(w_{3}-w_{5}\right) \gamma+w_{3}(\mu+\theta) \\
w_{4}^{\prime}(t)=-\frac{\partial H}{\partial Q}=\left(w_{4}-w_{5}\right) \varphi+w_{4}(\mu+\theta) \\
w_{5}^{\prime}(t)=-\frac{\partial H}{\partial R}=1+w_{5} \mu
\end{array}\right.
$$

with boundary conditions $w_{i}\left(t_{f}\right)=0, i=1, \ldots, 5$. Also, the optimal control variable $u^{*}$, subject to (26) and satisfying

$$
u^{*}=\max \left\{0, \min \left\{1, \frac{\left(w_{3}-w_{4}\right) I}{2 K}\right\}\right\}
$$


Remark 6. Note here that the optimality system contains state system (26), adjoint system (11) with its initial and boundary conditions, and the optimal control variable $u^{*}$ given by (30). Hence, the optimality system with quarantine intervention is expressed by

$$
\begin{aligned}
& S^{\prime}(t)=\Lambda-\frac{\beta S I}{1+\alpha I}-\mu S, \\
& E^{\prime}(t)=\frac{\beta S I}{1+\alpha I}-(\eta+\varepsilon+\mu) E, \\
& I^{\prime}(t)=\eta E-\left(u^{*}+\gamma+\mu+\theta\right) I, \\
& Q^{\prime}(t)=u^{*} I-(\varphi+\mu+\theta) Q \\
& R^{\prime}(t)=\varepsilon E+\gamma I+\varphi Q-\mu R, \\
& w_{1}^{\prime}(t)=-\frac{\partial H}{\partial S}=\frac{\left(w_{1}-w_{2}\right) \beta I}{1+\alpha I}+w_{1} \mu+\left(w_{1}-w_{5}\right) \psi \\
& w_{2}^{\prime}(t)=-\frac{\partial H}{\partial E}=-1+\left(w_{2}-w_{3}\right) \eta+\left(w_{2}-w_{5}\right) \varepsilon+w_{2} \mu \\
& w_{3}^{\prime}(t)=-\frac{\partial H}{\partial I}=-1+\frac{\left(w_{1}-w_{2}\right) \beta S}{(1+\alpha I)^{2}}+\left(w_{3}-w_{4}\right) u^{*}+\left(w_{3}-w_{5}\right) \gamma+w_{3}(\mu+\theta) \\
& w_{4}^{\prime}(t)=-\frac{\partial H}{\partial Q}=\left(w_{4}-w_{5}\right) \varphi+w_{4}(\mu+\theta), \\
& w_{5}^{\prime}(t)=-\frac{\partial H}{\partial R}=1+w_{5} \mu
\end{aligned}
$$

with initial conditions at time $t_{0}$ and boundary conditions, $w_{i}\left(t_{f}\right)=0, i=1, \ldots, 5$.

Please see Appendixes A.6 and A.7 for the proofs of Theorems 7 and 8. According to Lemma 4.1 in [37] and the proof of Theorem 2 in [38], the uniqueness of solution for the optimality system follows easily.

Theorem 9. The solution for the optimality system is unique for a small enough $t_{f}$.

We finally carry out a set of numerical experiments to validate the obtained-above control strategy. The modeling parameters and initial numbers are taken as those of Section 4.1. From Figure 5, the dynamics of the system without/with quarantine are presented as follows:
(1) Figure 5(a) shows the trend of the susceptible number without/with quarantine, where we see that the susceptible number will decay sharply because most of them become recovered with quarantine intervention.

(2) Figure 5(b) shows the trend of the exposed number without/with quarantine, which indicates that the exposed number with quarantine gets much smaller, and the device exposed to worms persists for a shorter time.

(3) Figure 5(c) shows the trend of the infectious number without/with quarantine. We observed that the infectious maximum number is nearly 8000 without quarantine, but this number is less than 500 with quarantine. 
(4) Figure 5(d) shows the trend of the recovered number without/with quarantine, which gives that with quarantine, a larger number of mobile devices become recovered more quickly.

5.2. Control Strategies Based on $R_{0}$. The basic reproduction number $R_{0}$, defined as (19), is a sharp threshold value that determines completely the dynamics of worm propagation and performs an important role in controlling the worm epidemic. As described in Theorems 2 and 3, the worm-free equilibrium point $P^{0}$ is stable locally and globally under condition that $R_{0} \leq 1$. Hence, we adopt the corresponding parameters such that $R_{0} \leq 1$ to prevent the worm epidemic. From equation (19), the number $R_{0}$ relates to the parameters as follows: the recruitment rate $\Lambda$, the quarantine rate $\xi$, the recovery rates $\psi, \varepsilon$, and $\gamma$, the infection rates $\beta$ and $\eta$, and the death rates $\mu$ and $\theta$.

According to Theorems 2 and 3, the following corollary regarding the parameters of $R_{0}$ is given to prevent the worm epidemic, and its proof is delayed to Appendix A.8.

Corollary 1. To control the epidemic of mobile networkbased worms, the following assertions for all the parameters in (19) should be true:

$$
\begin{aligned}
& \Lambda<\frac{(\psi+\mu)(\xi+\gamma+\theta+\mu)}{\beta} ; \\
& \beta<\frac{(\psi+\mu)(\xi+\gamma+\theta+\mu)}{\Lambda} ; \\
& \psi>\frac{\beta \eta \Lambda}{(\eta+\varepsilon+\mu)(\xi+\gamma+\theta+\mu)}-\mu \\
& \xi>\frac{\beta \eta \Lambda}{(\psi+\mu)(\eta+\varepsilon+\mu)}-(\gamma+\mu+\theta) \\
& \gamma>\frac{\beta \eta \Lambda}{(\psi+\mu)(\eta+\varepsilon+\mu)}-(\xi+\mu+\theta) \\
& \theta>\frac{\beta \eta \Lambda}{(\psi+\mu)(\eta+\varepsilon+\mu)}-(\xi+\gamma+\mu) \\
& \varepsilon>\frac{\beta \eta \Lambda}{(\psi+\mu)(\xi+\gamma+\theta+\mu)}-(\eta+\mu) \\
& \eta<\frac{(\psi+\mu)(\varepsilon+\mu)(\xi+\gamma+\theta+\mu)}{\beta \Lambda-(\psi+\mu)(\xi+\gamma+\theta+\mu)}, \quad \text { if } \beta \Lambda>(\psi+\mu)(\xi+\gamma+\theta+\mu) \text {; } \\
& \text { or } \eta>\frac{(\psi+\mu)(\varepsilon+\mu)(\xi+\gamma+\theta+\mu)}{\beta \Lambda-(\psi+\mu)(\xi+\gamma+\theta+\mu)}, \quad \text { if } \beta \Lambda<(\psi+\mu)(\xi+\gamma+\theta+\mu) \text {. }
\end{aligned}
$$

Considering the parameter values appearing in Section 4.1 except for $\psi$, we find out that the transition from the worm-free regime to the worm-existence one happens when $\psi=0.3573$, which is in line with the lower-bound of $\psi$, obtained by Corollary 1 . And the similar conclusions still hold for the other parameters.

By using the partial derivatives of $R_{0}$ in equation (19), it follows that 


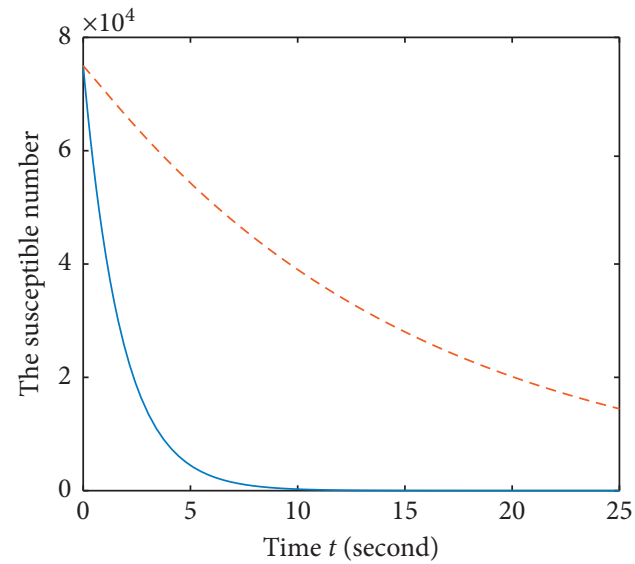

- - Without quarantine

_ With quarantine

(a)

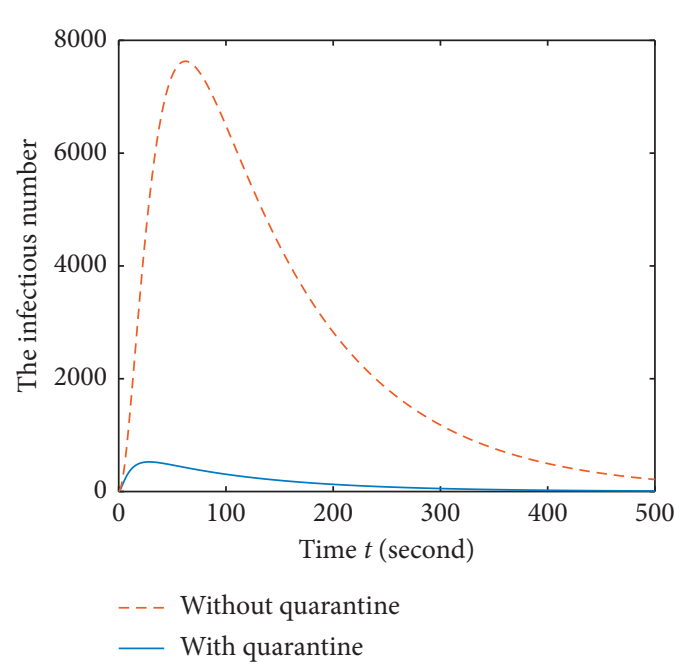

(c)

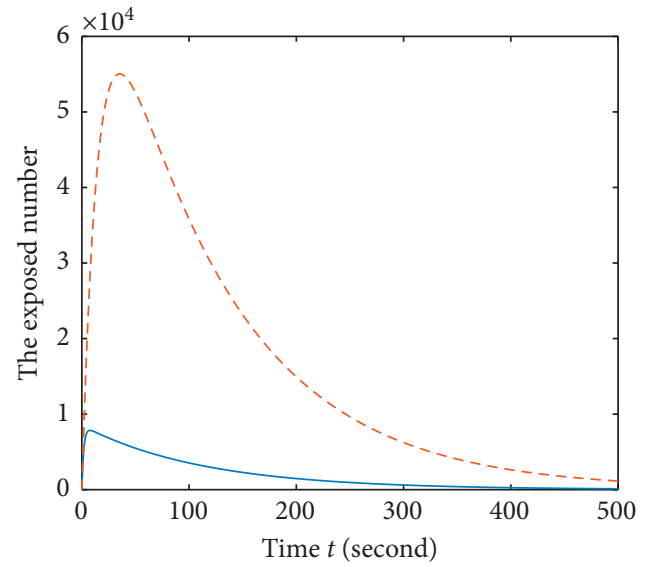

- - - Without quarantine

— With quarantine

(b)

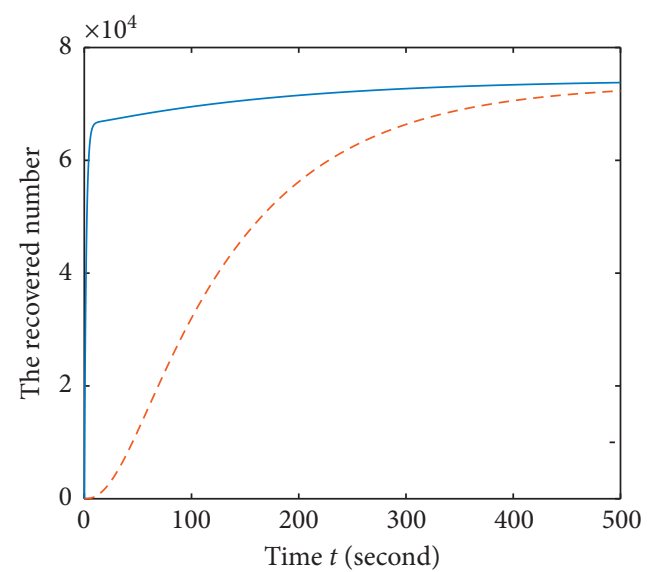

- - Without quarantine

_ With quarantine

FIgURE 5: Dynamics of system without/without quarantine.

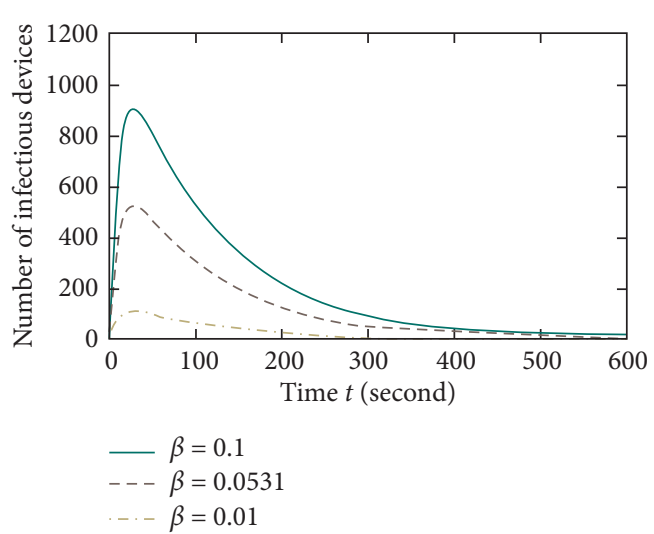

(a)

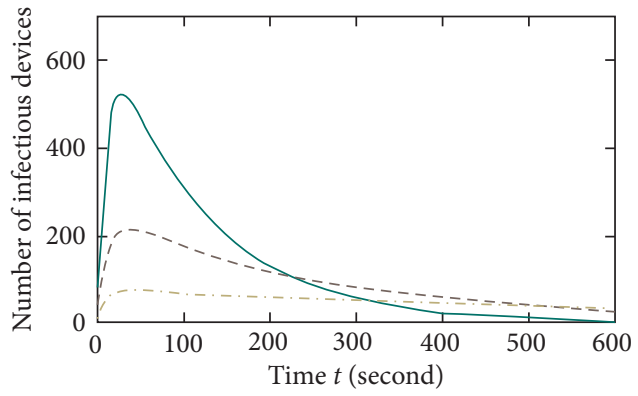

$\begin{aligned} \eta & =0.008 \\ --\eta & =0.003 \\ -\eta & =0.001\end{aligned}$

(b) 

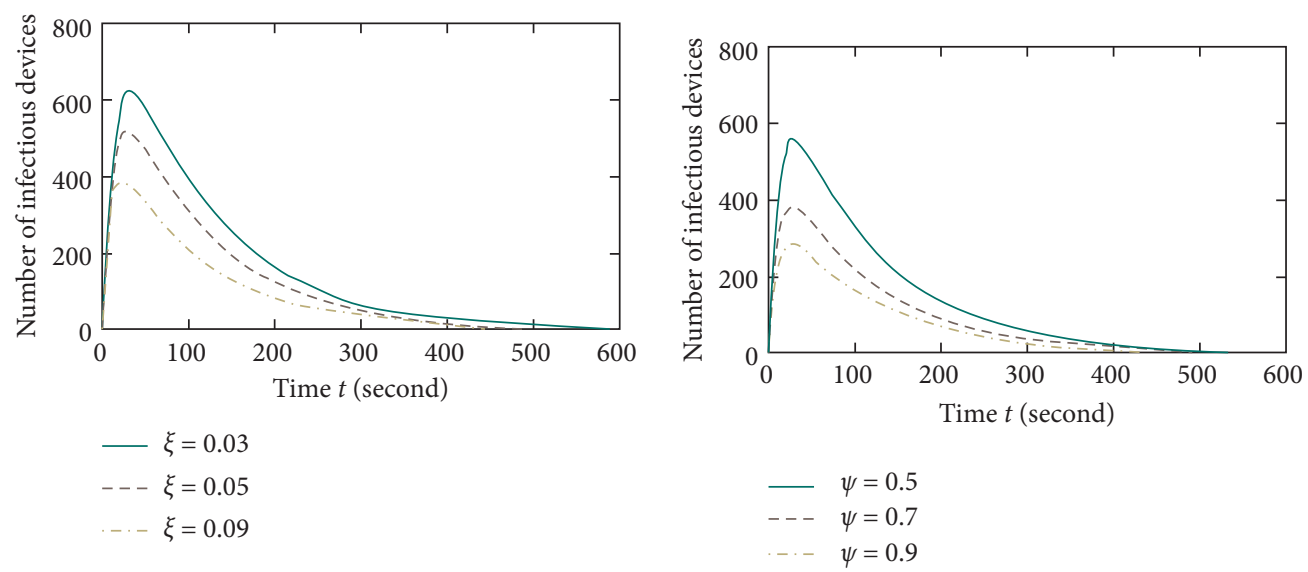

(c)

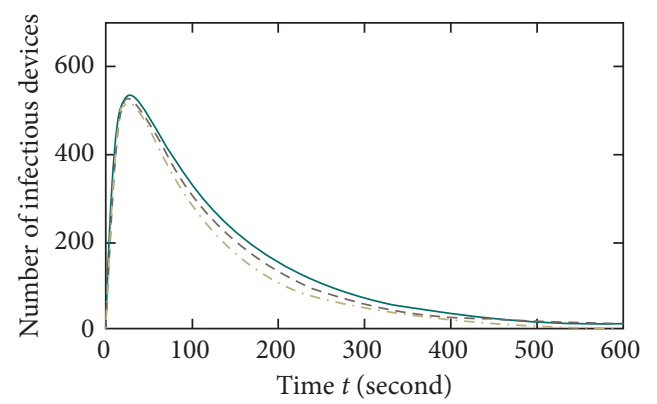

(d)

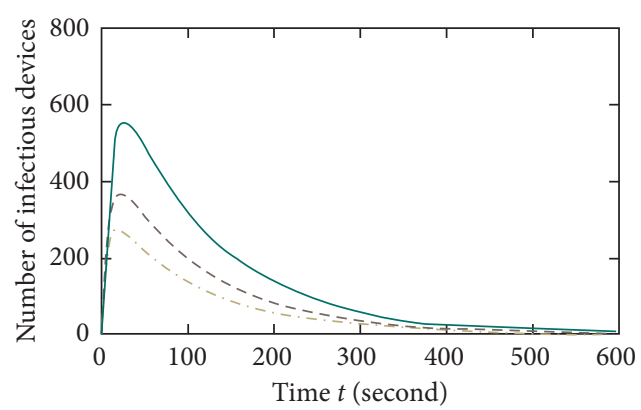

$\begin{aligned}-\epsilon & =0.0000 \\ ---\epsilon & =0.0008 \\ --\epsilon & =0.0018\end{aligned}$

$\gamma=0.05$

$--\gamma=0.10$

$\gamma=0.15$

(e)

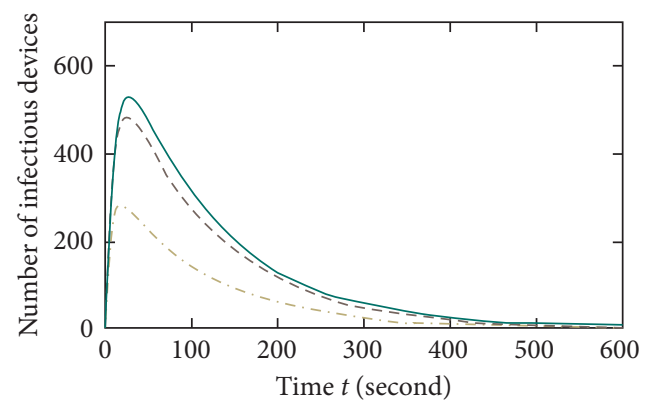

$\begin{aligned}-\theta & =0.00 \\ --\theta & =0.01 \\ -\theta & =0.09\end{aligned}$

(g)

FIGURE 6: Impacts of modeling parameters on the infectious devices number. 


$$
\begin{aligned}
& \frac{\partial R_{0}}{\partial \beta}>0, \\
& \frac{\partial R_{0}}{\partial \eta}>0, \\
& \frac{\partial R_{0}}{\partial \Lambda}>0, \\
& \frac{\partial R_{0}}{\partial \mu}<0, \\
& \frac{\partial R_{0}}{\partial \varepsilon}<0, \\
& \frac{\partial R_{0}}{\partial \psi}<0, \\
& \frac{\partial R_{0}}{\partial \xi}<0, \\
& \frac{\partial R_{0}}{\partial \gamma}<0, \\
& \frac{\partial R_{0}}{\partial \theta}<0 .
\end{aligned}
$$

Hence, if all the parameters of $R_{0}$, except for only one, are set to be constant, the function $R_{0}$ decreases as $\beta$, $\eta$, and $\Lambda$ decrease, or as $\mu, \varepsilon, \psi, \xi, \gamma$, and $\theta$ increase. This result, along with Theorems 2 and 3 , shows that to reduce the value of $R_{0}$ to be less than 1 , it suffices to decrease the values of $\beta, \eta$, and $\Lambda$, or increase the values of $\mu, \varepsilon, \xi, \gamma$, and $\theta$.

By the above analysis on $R_{0}$, a series of control strategies are established to control the epidemic of worms based on mobile networks, and a set of numerical experiments are conducted to demonstrate the effectiveness of obtained strategies, especially to illustrate the influences of modeling parameters on the infectious device number and the worm spreading speed, which, in fact, are two critical factors to judge the severity of worm epidemic:

(1) Decreasing the rate $\beta$ infected in group $S$ via using immunization procedures and/or antivirus software: conduct a numerical experiment to study the impact on the infectious number by different values $\beta=0.1$, 0.0531, and 0.01. As shown in Figure 6(a), the infectious device number and the worm spreading time both get smaller with the reduction of the infection rate $\beta$.

(2) Decreasing the infectious rate $\eta$ from group $E$ : set different values for $\eta$ as $0.008,0.003$, and 0.001 to consider its impact on the infectious number. The simulation result is shown from Figure 6(b), namely, that as the rate $\eta$ becomes smaller, the malicious worms will infect fewer devices in a longer period.

(3) Increasing the rate $\xi$ quarantined to group $Q$ via strengthening the capabilities of quarantine strategy: consider the effect on the worm propagation by $\xi=0.03,0.05$, and 0.09 . From Figure 6(c), we see that a larger rate of quarantine can lead to a small number of infectious cases, a lower speed of worm spreading, and a shorter time it takes to control the worm epidemic.

(4) Increasing the rate $\psi$ recovered from group $S$ via improving the quality and the user scale of antivirus software: Set $\psi=0.5,0.7$, and 0.9 , and from Figure $6(\mathrm{~d})$, we observe that as the rate $\psi$ increases, the infectious number will be smaller, the speed of worm propagation will become lower, and the time to control the epidemic of worm will get shorter.

(5) Increasing the rate $\varepsilon$ recovered from group $E$ via reminding to install antivirus software: consider the impact of rate $\varepsilon$ on the infectious number by $\varepsilon=0$, 0.0008 , and 0.0018 . From Figure 6(e), it follows that a larger rate $\varepsilon$ can result in reducing the size of mobile devices infected by the network-based worms.

(6) Increasing the rate $\gamma$ recovered from group $I$ via enhancing the efficacy of antiviral treatment: to analyze the impact of rate $\gamma$ on the number of infectious devices with $\gamma=0.05,0.10$, and 0.15 . See Figure 6(f), which indicates that with the increase of $\gamma$, the malicious worm will infect fewer mobile devices and spread in mobile networks for a shorter period.

(7) Increasing the rate $\theta$ of worm-related death via stopping and even eliminating infectious devices: set $\theta=0.001,0.01$, and 0.09 , and from Figure $6(\mathrm{~g})$, we show that as the rate $\theta$ becomes larger, the worm spreads more slowly, the epidemic lasts in a shorter term, and the mobile devices infected by this worm becomes fewer.

\section{Conclusions}

This paper investigates dynamics and control of worm epidemic based on mobile networks via a general SEIQR-type model with a saturated incidence rate, in which this rate can tend to its saturation level with the increase of the infectious number, and obviously, it is more realistic as it can present the crowding effect of infectious individuals and also guarantee the boundness of contact rate. The innovations of this paper are (a) to consider the recovery process from the susceptible group to the recovered group owing to sufficient defense mechanisms or/and antivirus treatments; (b) to discuss the dysfunction of devices related to worm-related death because the crash of most devices results from the attack of malicious worms; and (c) to establish the control strategies in defending against the epidemic of worms.

Our results suggest that the basic reproduction number $R_{0}$, as a sharp threshold value, can completely determine the dynamic behaviors of worm propagation and the control strategies for worm epidemic. More specifically, if $R_{0} \leq 1$, the worm-free equilibrium is asymptotically stable, and the epidemic of worm will eventually disappear and remain under control; if $R_{0}>1$, the asymptotical stability of wormexistence equilibrium is derived to imply that the epidemic will remain persistent and uncontrollable. This study gives new insights to mobile network security, namely, that is predicting the worm spreading tendency, identifying the 
epidemic control strategies, and estimating the worm popularity level. A series of numerical experiments are conducted to show the rationality of our obtained results.

With the use of Pontryagin's Minimum Principle [35], we devise the optimal control strategy to minimize the quarantine cost and the infected number as well as maximize the recovered number. Furthermore, by the analysis for $R_{0}$, we present a set of control strategies to fight against the epidemic of worm based on mobile networks, including to decrease the rates of infection from groups $S$ and $E$ and to increase the rate of quarantine, the rate of worm-related death, and the rates of recovery from groups $S, E$, and $I$.
Also, increasing the parameter $\alpha$ of saturated incidence rate can be used to stop the worm epidemic. Some numerical experiments are also implemented to validate the effectiveness of these obtained strategies.

\section{Appendix}

\section{A. Proofs of Theoretical Results}

A.1. Proof of Theorem 2. From (1) and (17), we obtain the Jacobian matrix at worm-free equilibrium $P^{0}$ as

$$
J\left(P^{0}\right)=\left(\begin{array}{ccccc}
-(\psi+\mu) & 0 & -\frac{\beta \Lambda}{\psi+\mu} & 0 & 0 \\
0 & -(\eta+\varepsilon+\mu) & \frac{\beta \Lambda}{\psi+\mu} & 0 & 0 \\
0 & \eta & -(\xi+\gamma+\mu+\theta) & 0 & 0 \\
0 & 0 & \xi & -(\varphi+\mu+\theta) & 0 \\
\psi & \varepsilon & \gamma & \varphi & -\mu
\end{array}\right) \text {, }
$$

and its characteristic equation is

$$
\begin{aligned}
\left|\lambda I-J\left(P^{0}\right)\right| & =\left|\begin{array}{ccccc}
\lambda+\psi+\mu & 0 & \frac{\beta \Lambda}{\psi+\mu} & 0 & 0 \\
0 & \lambda+\eta+\varepsilon+\mu & -\frac{\beta \Lambda}{\psi+\mu} & 0 & 0 \\
0 & -\eta & \lambda+\xi+\gamma+\mu+\theta & 0 & 0 \\
0 & 0 & -\xi & \lambda+\varphi+\mu+\theta & 0 \\
-\psi & -\varepsilon & -\gamma & -\varphi & \lambda+\mu
\end{array}\right| \\
& =(\lambda+\mu)(\lambda+\sigma v+\mu)(\lambda+\varepsilon+\mu+\theta)\left[(\lambda+\eta+\varepsilon+\mu)(\lambda+\xi+\gamma+\mu+\theta)-\frac{\beta \eta \Lambda}{\psi+\mu}\right] \\
& =0 .
\end{aligned}
$$

Hence, Jacobian matrix $J\left(P^{0}\right)$ has three negative eigenvalues $\lambda_{1}=-\mu, \lambda_{2}=-(\sigma \nu+\mu)$, and $\lambda_{3}=-(\varphi+\mu+\theta)$, and its other eigenvalues are the roots of the following equation: 


$$
\lambda^{2}+(2 \mu+\eta+\varepsilon+\xi+\gamma+\theta) \lambda+(\eta+\varepsilon+\mu)(\xi+\gamma+\mu+\theta)-\frac{\beta \eta \Lambda}{\psi+\mu}=0 .
$$

Under the condition that $R_{0}>1$, we have $(\eta+\varepsilon+\mu)(\xi+\gamma+\mu+\theta)-((\beta \eta \Lambda) /(\psi+\mu))<0$. So, equation (A.3) is of two positive and negative solutions, which implies that $P^{0}$ is an unstable point. On the other hand, if $R_{0} \leq 1$, we conclude using Hurwitz criterion [39] that the real parts of all solutions of (A.3) are negative, and then those of all eigenvalues of $J\left(P^{0}\right)$ are also negative. Therefore, by the stability theory [40], we prove that this equilibrium point $P^{0}$ is locally and asymptotically stable if $R_{0} \leq 1$.

A.2. Proof of Theorem 3. Note that, the Lyapunov function is formulated as

$$
L(E, I)=\eta E+(\eta+\mu+\varepsilon) I .
$$

Taking the derivative of $L(E, I)$, we have

$$
\begin{aligned}
\frac{\mathrm{d} L(E, I)}{\mathrm{d} t} & =\eta E^{\prime}(t)+(\eta+\varepsilon+\mu) I^{\prime}(t) \\
& =\eta\left[\frac{\beta S I}{1+\alpha I}-(\eta+\varepsilon+\mu) E\right]+(\eta+\varepsilon+\mu)[\eta E-(\xi+\gamma+\mu+\theta) I] \\
& =\left[\frac{\eta \beta S}{1+\alpha I}-(\eta+\varepsilon+\mu)(\xi+\gamma+\mu+\theta)\right] I \\
& \leq\left[\eta \beta S^{0}-(\eta+\varepsilon+\mu)(\xi+\gamma+\mu+\theta)\right] I \\
& =\frac{\beta \eta S^{0}}{R_{0}}\left(R_{0}-1\right) I,
\end{aligned}
$$

where the second last step is due to (8) and (17). Hence, if $R_{0} \leq 1,(\mathrm{~d} L(E, I) / \mathrm{d} t)$ is negative semidefinite.

Furthermore, $(\mathrm{d} L(E, I) / \mathrm{d} t)=0$ if $I=0$. Actually, if $(\mathrm{d} L(E, I) / \mathrm{d} t)=0$, then

$$
\left[\frac{\eta \beta S}{1+\alpha I}-(\eta+\varepsilon+\mu)(\xi+\gamma+\mu+\theta)\right] I=0,
$$

and thus either $I=0 \quad$ or $S=((\eta+\varepsilon+\mu)(\xi+\gamma+$ $\mu+\theta)(1+\alpha I)) / \beta \eta$. In the second case, we have that if $R_{0}<1$, then

$$
0=S^{\prime}(t)=\frac{\Lambda}{R_{0}}\left(R_{0}-1\right)-\frac{(\eta+\varepsilon+\mu)(\xi+\gamma+\mu+\theta)[\beta+\alpha(\psi+\mu)] I}{\beta \eta}<0,
$$

which is apparently a contradictory statement. In addition, if $I=0,0=I^{\prime}(t)=\eta E$ and further $E=0$.

As a result, if $R_{0} \leq 1$, the largest compact invariant set of $\left\{(E, I) \in \Omega \mid L^{\prime} t(E, I) n=q 0\right\}$ is a singleton with the origin point. Furthermore, by (8) and (17), we have that $\left.\lim _{t \rightarrow \infty}(S(t), E(t), I(t), Q(t)), R(t)\right)=P^{0}$. Based on the LaSalles invariance principle [41], we prove that $P^{0}$ is globally stable if $R_{0} \leq 1$.

If $R_{0}>1$, it follows that $(\mathrm{d} L(E, I) / \mathrm{d} t)>0$ for $S$ sufficiently close to $\Lambda /(\psi+\mu)$ except for when $E=I=0$. Solutions starting sufficiently close to $P^{0}$ leave a neighborhood of $P^{0}$ except for those on the invariant $S$-axis, on which (1) reduces to $S^{\prime}=\Lambda-(\psi+\mu) S$ and then $S(t) \longrightarrow \Lambda /(\psi+\mu)$ as $t \longrightarrow \infty$.

In fact, the assertion that the worm-free equilibrium $P^{0}$ is unstable if $R_{0}>1$ can also follow from the similar analysis of eigenvalue to that in Appendix A.1.

A.3. Proof of Theorem 4. From the instability of $P^{0}, P^{0} \in \partial \Omega$, and Theorem 4.3 in [42], we derive that system (1) has uniform persistence in $\Omega$ if $R_{0}>1$. 
A.4. Proof of Theorem 5. From (20) and (21), we obtain the Jacobian matrix of (1) at worm-existence equilibrium point $P^{*}$ as

$$
J\left(P^{*}\right)=\left(\begin{array}{ccccc}
-\frac{\beta I^{*}}{1+\alpha I^{*}}-(\psi+\mu) & 0 & -\frac{\beta S^{*}}{\left(1+\alpha I^{*}\right)^{2}} & 0 & 0 \\
\frac{\beta I^{*}}{1+\alpha I^{*}} & -(\eta+\varepsilon+\mu) & \frac{\beta S^{*}}{\left(1+\alpha I^{*}\right)^{2}} & 0 & 0 \\
0 & \eta & -(\xi+\gamma+\mu+\theta) & 0 & 0 \\
0 & 0 & \xi & -(\varphi+\mu+\theta) & 0 \\
\psi & \varepsilon & \gamma & \varphi & -\mu
\end{array}\right) \text {, }
$$

and its characteristic equation as

$$
\begin{aligned}
& \left|\lambda \mathbf{I}-J\left(P^{*}\right)\right|=\left|\begin{array}{ccccc}
\lambda+\frac{\beta I^{*}}{1+\alpha I^{*}}+\psi+\mu & 0 & \frac{\beta S^{*}}{\left(1+\alpha I^{*}\right)^{2}} & 0 & 0 \\
-\frac{\beta I^{*}}{1+\alpha I^{*}} & \lambda+\eta+\varepsilon+\mu & -\frac{\beta S^{*}}{\left(1+\alpha I^{*}\right)^{2}} & 0 & 0 \\
0 & -\eta & \lambda+\xi+\gamma+\mu+\theta & 0 & 0 \\
0 & 0 & -\xi & \lambda+\varphi+\mu+\theta & 0 \\
-\psi & -\varepsilon & -\gamma & -\varphi & \lambda+\mu
\end{array}\right| \\
& =(\lambda+\mu)(\lambda+\varphi+\mu+\theta)\left\{(\lambda+\eta+\varepsilon+\mu)(\lambda+\xi+\gamma+\mu+\theta)\left[\lambda+\psi+\mu+\frac{\beta I^{*}}{1+\alpha I^{*}}\right]\right. \\
& \left.-\frac{\beta \eta S^{*}}{\left(1+\alpha I^{*}\right)^{2}}\left[\lambda+\psi+\mu+\frac{\beta I^{*}}{1+\alpha I^{*}}\right]+\frac{\beta^{2} \eta S^{*} I^{*}}{\left(1+\alpha I^{*}\right)^{3}}\right\} \\
& =0 \text {. }
\end{aligned}
$$

Thus, the matrix $J\left(P^{*}\right)$ has two negative eigenvalues $\lambda_{1}=-\mu$, and $\lambda_{2}=-(\varphi+\mu+\theta)$, and its other eigenvalues are given by the following equation: 


$$
\lambda^{3}+B_{1} \lambda^{2}+B_{2} \lambda+B_{3}=0, \quad \text { (A.10) where }
$$

$$
\begin{aligned}
& B_{1}=\eta+\varepsilon+\xi+\gamma+\theta+\psi+3 \mu+\frac{\beta I^{*}}{1+\alpha I^{*}}, \\
& B_{2}=(\eta+\varepsilon+\xi+\gamma+\theta+2 \mu)\left[\psi+\mu+\frac{\beta I^{*}}{1+\alpha I^{*}}\right]+(\eta+\varepsilon+\mu)(\xi+\gamma+\mu+\theta)-\frac{\beta \eta S^{*}}{\left(1+\alpha I^{*}\right)^{2}}, \\
& B_{3}=\frac{\beta I^{*}}{1+\alpha I^{*}}(\eta+\varepsilon+\mu)(\xi+\gamma+\mu+\theta)+(\psi+\mu)\left[(\eta+\varepsilon+\mu)(\xi+\gamma+\mu+\theta)-\frac{\beta \eta S^{*}}{\left(1+\alpha I^{*}\right)^{2}}\right] .
\end{aligned}
$$

Obviously, $B_{1}>0$, and since

$$
\frac{\beta \eta S^{*}}{1+\alpha I^{*}}=(\eta+\varepsilon+\mu)(\xi+\gamma+\mu+\theta),
$$

we get that $B_{2}>0$ and $B_{3}>0$. After a direct computation, we have $B_{1} B_{2}-B_{3}>0$. Hence, we have used the Hurwitz criterion [39] that the real parts of all solutions of (A.10) are negative, and then those of all eigenvalues of $J\left(P^{*}\right)$ are still negative. Therefore, we derive using the stability theory [40] that $P^{*}$ is locally stable if $R_{0}>1$.

A.5. Proof of Theorem 6. We firstly consider the subsystem of (1) as

$$
\left\{\begin{array}{l}
S^{\prime}(t)=\Lambda-\frac{\beta S I}{1+\alpha I}-(\psi+\mu) S \\
E^{\prime}(t)=\frac{\beta S I}{1+\alpha I}-(\eta+\varepsilon+\mu) E \\
I^{\prime}(t)=\eta E-(\xi+\gamma+\mu+\theta) I
\end{array}\right.
$$

Hence, the Jacobian matrix of system (A.3) is

$$
J=\left(\begin{array}{ccc}
-\frac{\beta I}{1+\alpha I}-(\psi+\mu) & 0 & -\frac{\beta S}{(1+\alpha I)^{2}} \\
\frac{\beta I}{1+\alpha I} & -(\eta+\varepsilon+\mu) & \frac{\beta S}{(1+\alpha I)^{2}} \\
0 & \eta & -(\xi+\gamma+\mu+\theta)
\end{array}\right) \text {, }
$$

$$
J^{[2]}=\left(\begin{array}{ccc}
-\frac{\beta I}{1+\alpha I}-m & \frac{\beta S}{(1+\alpha I)^{2}} & \frac{\beta S}{(1+\alpha I)^{2}} \\
\eta & -\frac{\beta I}{1+\alpha I}-n & 0 \\
0 & \frac{\beta I}{1+\alpha I} & -k
\end{array}\right) \text {, }
$$

where $m=\psi+\eta+\varepsilon+2 \mu, \quad n=\psi+\xi+\gamma+\theta+2 \mu$, and $k=\eta+\varepsilon+\xi+\gamma+\theta+2 \mu$.

Let $A=A(S, E, I)=\operatorname{diag}(1,(E / I),(E / I))$. Then, the directional derivative along $(S, E, I)$ is

$$
A_{f}=\operatorname{diag}\left(0, \frac{E^{\prime} I-I^{\prime} E}{I^{2}}, \frac{E^{\prime} I-I^{\prime} E}{I^{2}}\right) .
$$

Furthermore, we have

$$
\begin{aligned}
A_{f} A^{-1} & =\operatorname{diag}\left(0, \frac{E^{\prime}}{E}-\frac{I^{\prime}}{I}, \frac{E^{\prime}}{E}-\frac{I^{\prime}}{I}\right), \\
A J^{[2]} A^{-1} & =\left(\begin{array}{ccc}
-\frac{\beta I}{1+\alpha I}-m & \frac{\beta S I}{E(1+\alpha I)^{2}} & \frac{\beta S I}{E(1+\alpha I)^{2}} \\
\frac{\eta E}{I} & -\frac{\beta I}{1+\alpha I}-n & 0 \\
0 & \frac{\beta I}{1+\alpha I} & -k
\end{array}\right) .
\end{aligned}
$$

Let $B=A_{f} A^{-1}+A J^{[2]} A^{-1}$, which has its matrix form:

$$
B=\left(\begin{array}{ll}
B_{11} & B_{12} \\
B_{21} & B_{22}
\end{array}\right),
$$

where

and its second additive compound matrix is 


$$
\begin{aligned}
& B_{11}=-\frac{\beta I}{1+\alpha I}-m, \\
& B_{12}=\left(\frac{\beta S I}{E(1+\alpha I)^{2}}, \frac{\beta S I}{E(1+\alpha I)^{2}}\right), \\
& B_{21}=\left(\frac{\eta E}{I}, 0\right)^{T}, \\
& B_{22}=\left(\begin{array}{cc}
\frac{E^{\prime}}{E}-\frac{I^{\prime}}{I}-\frac{\beta I}{1+\alpha I}-n & 0 \\
\frac{\beta I}{1+\alpha I} & \frac{E^{\prime}}{E}-\frac{I^{\prime}}{I}-k
\end{array}\right) .
\end{aligned}
$$

Denote a vector in $\mathbb{R}^{3}$ by $(u, v, w)$ with its norm as follows:

$$
\|(u, v, w)\|=\max \{|u|,|v|+|w|\} .
$$

Let $\mu(B)$ be the Lozinski $\breve{l}$ measure with respect to this norm. As was stated by [43], we choose

$$
\mu(B) \leq \sup \left\{g_{1}, g_{2}\right\}
$$

where $g_{1}=\mu_{1}\left(B_{11}\right)+\left|B_{12}\right|, g_{2}=\left|B_{21}\right|+\mu_{1}\left(B_{22}\right)$, and $\left|B_{12}\right|$, $\left|B_{21}\right|$ are matrix norms with respect to the $l_{1}$ vector norm, and $\mu_{1}$ is the .. measure with respect to $l_{1}$ norm. Thus, it follows that

$$
\begin{aligned}
\mu_{1}\left(B_{11}\right) & =-\frac{\beta I}{1+\alpha I}-m, \\
\left|B_{21}\right| & =\frac{\eta E}{I} \\
\left|B_{12}\right| & =\frac{\beta S I}{E(1+\alpha I)^{2}}, \\
\mu_{1}\left(B_{22}\right) & =\max \left\{\frac{E^{\prime}}{E}-\frac{I^{\prime}}{I}-n, \frac{E^{\prime}}{E}-\frac{I^{\prime}}{I}-k\right\} \\
& =\frac{E^{\prime}}{E}-\frac{I^{\prime}}{I}-\min \{n, k\} .
\end{aligned}
$$

Consequently,

$$
\begin{aligned}
& g_{1}=-\frac{\beta I}{1+\alpha I}-m+\frac{\beta S I}{E(1+\alpha I)^{2}}, \\
& g_{2}=\frac{\eta E}{I}+\frac{E^{\prime}}{E}-\frac{I^{\prime}}{I}-\min \{n, k\} .
\end{aligned}
$$

By (A.3), we have

$$
\begin{aligned}
& \frac{E^{\prime}}{E}=\frac{\beta S I}{E(1+\alpha I)}-(\eta+\varepsilon+\mu), \\
& \frac{I^{\prime}}{I}=\frac{\eta E}{I}-(\xi+\gamma+\mu+\theta) .
\end{aligned}
$$

Then,

$$
\begin{aligned}
g_{1} & =-\frac{\beta I}{1+\alpha I}-m+\frac{\beta S I}{E(1+\alpha I)}+\left[\frac{\beta S I}{E(1+\alpha I)^{2}}-\frac{\beta S I}{E(1+\alpha I)}\right] \\
& =\frac{E^{\prime}}{E}-\mu-\frac{\beta S I}{(1+\alpha I)}-\psi+\left[\frac{\beta S I}{E(1+\alpha I)^{2}}-\frac{\beta S I}{E(1+\alpha I)}\right] \\
& \leq \frac{E^{\prime}}{E}-\mu, \\
g_{2} & =\frac{E^{\prime}}{E}+(\xi+\gamma+\mu+\theta)-\min \{n, k\} \\
& =\frac{E^{\prime}}{E}-\mu \\
& \leq \frac{E^{\prime}}{E}-\mu .
\end{aligned}
$$

Furthermore, we have

$$
\mu(B) \leq \sup \left\{g_{1}, g_{2}\right\} \leq \frac{E^{\prime}}{E}-\mu .
$$

Since there is a sufficiently large $T>0$ such that for all $t>T,(E(t) / E(0))<e^{\mu t / 2}$, i.e., $(1 / t) \ln (E(t) / E(0))<(\mu / 2)$, we show that, for all $t>T$,

$$
\frac{1}{t} \int_{0}^{t} \mu(B) \mathrm{d} s \leq \frac{1}{t} \int_{0}^{t}\left(\frac{E^{\prime}}{E}-\mu\right) \mathrm{d} s=\frac{1}{t} \ln \frac{E(t)}{E(0)}-\mu<-\frac{\mu}{2},
$$

which yields that

$$
q=\limsup _{t \longrightarrow \infty} \sup _{(V(0), E(0), I(0)) \in \operatorname{int}(\Omega)} \frac{1}{t} \int_{0}^{t} \mu(B) \mathrm{d} s \leq-\frac{\mu}{2}<0 .
$$

Therefore, we apply the Li-Muldowney geometric approach (i.e., Theorem 3.5 of [28]) to know that $\left(S^{*}, E^{*}, I^{*}\right)$ is globally stable.

Subsequently, we turn to the subsystem of (1) as

$$
\left\{\begin{array}{l}
Q^{\prime}(t)=\xi I-(\varphi+\mu+\theta) Q \\
R^{\prime}(t)=\psi S+\varepsilon E+\gamma I+\varphi Q-\mu R
\end{array}\right.
$$

which has its limit system as

$$
\left\{\begin{array}{l}
Q^{\prime}(t)=\xi I^{*}-(\varphi+\mu+\theta) Q \\
R^{\prime}(t)=\psi S^{*}+\varepsilon E^{*}+\gamma I^{*}+\varphi Q-\mu R .
\end{array}\right.
$$

By a simple computation, we have

$$
\left\{\begin{array}{l}
Q(t)=e^{-(\varphi+\mu+\theta) t}\left[Q(0)+\xi I^{*} \int_{0}^{t} e^{(\varphi+\mu+\theta) s} \mathrm{~d} s\right], \\
R(t)=e^{-\mu t}\left[S(0)+\int_{0}^{t}\left[\psi V^{*}+\varepsilon E^{*}+\gamma I^{*}+\varphi Q(s)\right] e^{\mu s} \mathrm{~d} s\right],
\end{array}\right.
$$

which proves that 


$$
Q(t) \longrightarrow \frac{\xi I^{*}}{\varphi+\mu+\theta}=Q^{*}, R(t) \longrightarrow \frac{\psi V^{*}+\varepsilon E^{*}+\gamma I^{*}+\varphi Q^{*}}{\mu}=R^{*}, \quad \text { as } t \longrightarrow \infty \text {. }
$$

As a result, we show that this equilibrium point $P^{*}$ is globally stable if $R_{0}>1$.

A.6. Proof of Theorem 7. Firstly, we need to check the following conditions:

(1) State system (26) has bounded coefficient; then, the set of control variables and state is nonempty.

(2) Due to the boundedness of the solutions of (26), the control set $U$ is closed and convex.

(3) The right-hand side of (26) is bounded by a linear function in the state and control variables.

(4) The integrand, $K u^{2}(t)+E(t)+I(t)-R(t)$, of the cost function is convex on $U$.

(5) There are $c_{1}>0, c_{2}>0$, and $\pi>1$ such that

$$
K u^{2}(t)+E(t)+I(t)-R(t) \geq c_{1}|u|^{\pi}-c_{2},
$$

where $c_{2}$ relies on the boundedness of $S(t), E(t)$, and $I(t)$, and $c_{1}>0$ since $K>0$ and $L>0$.

Then, by Theorem 4.1 of Fleming and Rishel [36], the existence of optimal control pair follows immediately.

A.7. Proof of Theorem 8. According to Pontryagin's Minimum Principle [35], the adjoint system and its boundary conditions have standard forms. By the differentiating Hamiltonian (29), we get the adjoint system as (11) with $w_{i}\left(t_{f}\right)=0, i=1, \ldots, 5$.

Under the optimal conditions, we have that the optimal control variable, $u^{*}$, satisfies

$$
\frac{\partial H}{\partial u^{*}}=0
$$

Note that, the Hamiltonian (29) can be expressed as

$H=K u^{2}+\left(w_{4}-w_{3}\right) I u+$ other terms without $u$,

which, along with (A.34), implies that

$$
\frac{\partial H}{\partial u^{*}}=2 K u^{*}+\left(w_{4}-w_{3}\right) I=0 .
$$

Then, it follows that

$$
u^{*}=\frac{\left(w_{3}-w_{4}\right) I}{2 K}
$$

Because the standard control is bounded, we obtain that

$$
u^{*}= \begin{cases}0, & \frac{\left(w_{3}-w_{4}\right) I}{2 K} \leq 0 \\ \frac{\left(w_{3}-w_{4}\right) I}{2 K}, & 0<\frac{\left(w_{3}-w_{4}\right) I}{2 K}<1 \\ 1, & \frac{\left(w_{3}-w_{4}\right) I}{2 K} \geq 1 .\end{cases}
$$

Hence, the optimal control variable $u^{*}$ has the compact form as (30), which completes this proof.

A.8. Proof of Corollary 1. By (19), we know that $R_{0}<1$ if

$$
\frac{\beta \eta \Lambda}{(\psi+\mu)(\eta+\varepsilon+\mu)(\xi+\gamma+\mu+\theta)}<1 .
$$

Hence by Theorems 2 and 3, the epidemic of worm does not outbreak if

$$
\beta \eta \Lambda<(\psi+\mu)(\eta+\varepsilon+\mu)(\xi+\gamma+\mu+\theta) .
$$

Since $\eta<\eta+\varepsilon+\mu$, we have $R_{0}<1$ if

$$
\Lambda<\frac{(\psi+\mu)(\xi+\gamma+\mu+\theta)}{\beta} \text {. }
$$

Likewise, the corresponding assertions of other modeling parameters can also be obtained.

\section{Data Availability}

No data were used to support the study.

\section{Conflicts of Interest}

The authors declare that they have no conflicts of interest.

\section{Acknowledgments}

The research was supported by the National Natural Science Foundation of China (no. 11871289) and the Humanities and Social Sciences Foundation of the Ministry of Education of China (no. 20YJCZH034).

\section{References}

[1] J. Scharr, "New WiFi worm can spread like an airborne disease," Mar, vol. 3, 2014, http://wwww.yahoo.com/tech/new-wifiworm-can-read-like-an-airborne-disease-78496514830.html..

[2] S. Peng, "A survey on malware containment models in smartphones," Applied Mechanics and Materials, vol. 263-266, pp. 3005-3011, 2013. 
[3] K. Haldar and B. K. Mishra, "A mathematical model for a distributed attack on targeted resources in a computer network," Communications in Nonlinear Science and Numerical Simulation, vol. 19, no. 9, pp. 3149-3160, 2014.

[4] J. D. Hernndez Guilln, A. Martn del Rey, and L. Hernndez Encinas, "Study of the stability of a SEIRS model for computer worm propagation," Physica A: Statistical Mechanics and its Applications, vol. 479, pp. 411-421, 2017.

[5] J. D. Hernndez Guilln and A. Martn del Rey, "Modeling malware propagation using a carrier compartment," Communications in Nonlinear Science and Numerical Simulation, vol. 56, pp. 217-226, 2018.

[6] T. Kuniya, H. Inaba, and J. Yang, "Global behavior of SIS epidemic models with age structure and spatial heterogeneity," Japan Journal of Industrial and Applied Mathematics, vol. 35, no. 2, pp. 669-706, 2018.

[7] O. A. Toutonji, S. M. Yoo, and M. Park, "Stability analysis of VEISV propagation modeling for network worm attack," Applied Mathematical Modelling, vol. 36, pp. 751-2761, 2012.

[8] Q. Zhu, X. Yang, and J. Ren, "Modeling and analysis of the spread of computer virus," Communications in Nonlinear Science and Numerical Simulation, vol. 17, no. 12, pp. 51175124, 2012.

[9] S. Pulvirenti, R. Maria Stefania Costa, and P. Pavone, "Francesco Cupani: the "scientific network" of his time and the making of the Linnaean "system," Acta Botanica Gallica, vol. 162, no. 3, pp. 215-223, 2015.

[10] G. Ferrauto, R. M. S. Costa, P. Pavone, and G. L. Cantarella, "Human impact assessment on the Sicilian agroecosystems through the evaluation of melliferous areas," Annali Di Botanica, vol. 3, pp. 237-244, 2013.

[11] Y. Han, "A tripartite evolutionary game analysis of enterprises' behaviour in the platform ecosystem," Discrete Dynamics in Nature and Society, vol. 2020, Article ID 8256091, 10 pages, 2020.

[12] X. Liu and L. Yang, "Stability analysis of an SEIQV epidemic model with saturated incidence rate," Nonlinear Analysis: Real World Applications, vol. 13, no. 6, pp. 2671-2679, 2012.

[13] C. Sun and Y.-H. Hsieh, "Global analysis of an SEIR model with varying population size and vaccination," Applied Mathematical Modelling, vol. 34, no. 10, pp. 2685-2697, 2010.

[14] B. Buonomo, A. d'Onofrio, and D. Lacitignola, "Globally stable endemicity for infectious diseases with informationrelated changes in contact patterns," Applied Mathematics Letters, vol. 25, no. 7, pp. 1056-1060, 2012.

[15] L.-M. Cai and X.-Z. Li, "Analysis of a SEIV epidemic model with a nonlinear incidence rate," Applied Mathematical Modelling, vol. 33, no. 7, pp. 2919-2926, 2009.

[16] K. Chakraborty, S. Jana, and T. K. Kar, "Global dynamics and bifurcation in a stage structured prey-predator fishery model with harvesting," Applied Mathematics and Computation, vol. 218, no. 18, pp. 9271-9290, 2012.

[17] T. K. Kar and P. K. Mondal, "Global dynamics and bifurcation in delayed SIR epidemic model," Nonlinear Analysis: Real World Applications, vol. 12, no. 4, pp. 2058-2068, 2011.

[18] G. $\mathrm{Lu}$ and Z. Lu, "Geometric approach to global asymptotic stability for the SEIRS models in epidemiology," Nonlinear Analysis: Real World Applications, vol. 36, pp. 20-43, 2017.

[19] C. Sun, Y. Lin, and S. Tang, "Global stability for an special SEIR epidemic model with nonlinear incidence rates," Chaos, Solitons \& Fractals, vol. 33, no. 1, pp. 290-297, 2007.

[20] L. Wang, Z. Liu, and X. Zhang, "Global dynamics of an SVEIR epidemic model with distributed delay and nonlinear incidence," Applied Mathematics and Computation, vol. 284, pp. 47-65, 2016.

[21] X. Zhou, X. Shi, and J. Cui, "Stability and backward bifurcation on a cholera epidemic model with saturated recovery rate," Mathematical Methods in the Applied Sciences, vol. 40, no. 4, pp. 1288-1306, 2017.

[22] X. Xiao, P. Fu, C. Dou, Q. Li, G. Hu, and S. Xia, "Design and analysis of SEIQR worm propagation model in mobile internet," Communications in Nonlinear Science and Numerical Simulation, vol. 43, pp. 341-350, 2017.

[23] O. Diekmann, J. A. P. Heesterbeek, and J. A. J. Metz, "On the definition and the computation of the basic reproduction ratio $R_{0}$ in models for infectious diseases in heterogeneous populations," Journal of Mathematical Biology, vol. 28, no. 4, pp. 365-382, 1990.

[24] C. Castillo-Chavez, Z. Feng, and W. Huang, "On the computation of $R_{0}$ and its role on global stability," in Mathematical Approaches for Emerging and Reemerging Infectious Diseases: An Introduction, pp. 229-250, Springer, Berlin, Germany, 2002.

[25] X. Zhao, Dynamical Systems in Population Biology, Springer, New York, NY, USA, CMS Books in Mathematics, Canadian Mathematical Society, 2003.

[26] G. Butler, H. I. Freedman, and P. Waltman, "Uniformly persistent systems," Proceedings of the American Mathematical Society, vol. 96, no. 3, p. 425, 1986.

[27] G. Butler and P. Waltman, "Persistence in dynamical systems," Journal of Differential Equations, vol. 63, no. 2, pp. 255-263, 1986.

[28] M. Y. Li and J. S. Muldowney, "A geometric approach to global-stability problems," SIAM Journal on Mathematical Analysis, vol. 27, no. 4, pp. 1070-1083, 1996.

[29] Q. Gao and J. Zhuang, "Stability analysis and control strategies for worm attack in mobile networks via a VEIQS propagation model," Applied Mathematics and Computation, vol. 368, Article ID 124584, 2020.

[30] D. Moore, V. Paxson, S. Savage, C. Shannon, S. Staniford, and N. Weaver, "Inside the slammer worm," IEEE Security \& Privacy, vol. 1, no. 4, pp. 33-39, 2003.

[31] F. Wang, Y. Zhang, C. Wang, J. Ma, and S. Moon, "Stability analysis of a SEIQV epidemic model for rapid spreading worms," Computers \& Security, vol. 29, no. 4, pp. 410-418, 2010.

[32] O. Toutonji and S. M. Yoo, "Passive benign worm propagation modeling with dynamic quarantine defense," KSII Transactions on Internet and Information Systems, vol. 3, no. 1, pp. 96-107, 2009.

[33] A. K. Roy, P. K. Roy, and E. Grigorieva, "Mathematical insights on psoriasis regulation: role of Th1 and Th2 cells," Mathematical Biosciences and Engineering, vol. 15, no. 3, pp. 717-738, 2018.

[34] F. Al Basir, K. B. Blyuss, and S. Ray, "Modelling the effects of awareness-based interventions to control the mosaic disease of Jatropha curcas," Ecological Complexity, vol. 36, pp. 92-100, 2018.

[35] L. S. Pontryagin, V. G. Boltyanskii, R. V. Gamkrelidze, and E. F. Mishchenko, Mathematical Theory of Optimal Processes, Gordon and Breach Science Publishers, London, UK, 1986.

[36] W. H. Fleming and R. W. Rishel, Deterministic and Stochastic Optimal Control, Springer-Verlag, Berlin, Germany, 1975.

[37] H. R. Joshi, "Optimal control of an HIV immunology model," Optimal Control Application and Methods, vol. 23, pp. 199213, 2002. 
[38] A. N. Chatterjee and P. K. Roy, "Anti-viral drug treatment along with immune activator IL-2: a control-based mathematical approach for HIV infection," International Journal of Control, vol. 85, no. 2, pp. 220-237, 2012.

[39] A. Hurwitz, "On the conditions under which an equation has only roots with negative real parts," in Selected Papers on Mathematical Trends in Control Theory, R. T. Ballman, Ed., Dover, New York, NY, USA, 1964.

[40] R. C. Robinson, An Introduction to Dynamical System: Continuous and Discrete, Prentice-Hall, Upper Saddle River, NJ, USA, 2004.

[41] J. P. LaSalle, The Stability of Dynamical Systems, Regional Conference Series in Applied Mathematics, SIAM, Philadelphia, PA, USA, 1976.

[42] H. I. Freedman, S. Ruan, and M. Tang, "Uniform persistence and flows near a closed positively invariant set," Journal of Dynamics and Differential Equations, vol. 6, no. 4, pp. 583600, 1994.

[43] R. H. Martin, "Logarithmic norms and projections applied to linear differential systems," Journal of Mathematical Analysis and Applications, vol. 45, no. 2, pp. 432-454, 1974. 